\title{
Nonlinear Unsteady Aerodynamic Modeling Using Wind Tunnel and Computational Data
}

\author{
Patrick C. Murphy ${ }^{1}$ \\ NASA Langley Research Center, Hampton, VA, 23681-2199 \\ Vladislav Klein ${ }^{2}$ \\ The George Washington University, NASA Langley Research Center, Hampton, VA, 23681-2199 \\ and \\ Neal T. Frink ${ }^{3}$ \\ NASA Langley Research Center, Hampton, VA, 23681-2199
}

\begin{abstract}
Extensions to conventional aircraft aerodynamic models are required to adequately predict responses when nonlinear unsteady flight regimes are encountered, especially at high incidence angles and under maneuvering conditions. For a number of reasons, such as loss of control, both military and civilian aircraft may extend beyond normal and benign aerodynamic flight conditions. In addition, military applications may require controlled flight beyond the normal envelope, and civilian flight may require adequate recovery or prevention methods from these adverse conditions. These requirements have led to the development of more general aerodynamic modeling methods and provided impetus for researchers to improve both techniques and the degree of collaboration between analytical and experimental research efforts. In addition to more general mathematical model structures, dynamic test methods have been designed to provide sufficient information to allow model identification. This paper summarizes research to develop a modeling methodology appropriate for modeling aircraft aerodynamics that include nonlinear unsteady behaviors using both experimental and computational test methods. This work was done at Langley Research Center, primarily under the NASA Aviation Safety Program, to address aircraft loss of control, prevention, and recovery aerodynamics.
\end{abstract}

\section{Nomenclature}

\begin{tabular}{|c|c|c|c|}
\hline$A, B, C$ & $=$ transfer function coefficients & $f$ & $=$ frequency, $\mathrm{Hz}$ \\
\hline$A_{j}, B_{j}$ & $=$ Fourier coefficients & $J(\theta)$ & $=$ sum of squared residuals \\
\hline$a, b_{1}$ & $=$ indicial function parameters & $k$ & $=$ reduced frequency, $2 \pi f \ell / V$ \\
\hline$b$ & $=$ wing span, $\mathrm{ft}$ & $\ell$ & $=$ half chord or half span, $\mathrm{ft}$ \\
\hline$C_{a}$ & aero coefficients, $a=[N, m, l, n, Y, A]$ & $m$ & $=$ No. of harmonics in Fourier expansion \\
\hline$C_{l}$ & rolling-moment coefficient & $N$ & number of data points \\
\hline$C_{N}$ & normal-force coefficient & $p, q, r$ & roll, pitch, and yaw rates, rad/sec \\
\hline $\bar{c}$ & mean aerodynamic chord, ft & $R^{2}$ & coefficient of determination \\
\hline $\bar{C}_{a_{\alpha}}, \bar{C}_{a_{\beta}}$ & in-phase components of $C_{a}$ & $s$ & estimated standard error \\
\hline $\bar{C}_{a_{q}}, \bar{C}_{a_{p}}$ & $=$ out-of-phase components of $C_{a}$ & $\begin{array}{l}t \\
V\end{array}$ & $\begin{array}{l}=\text { time, sec } \\
=\text { airspeed, } \mathrm{ft} / \mathrm{sec}\end{array}$ \\
\hline$D$ & $=$ time domain differential operator & $y$ & $=$ output variable \\
\hline$d_{0}, d_{1}$ & $=$ coefficients in two-step regression & $Z$ & $=$ measured variable \\
\hline
\end{tabular}

\footnotetext{
${ }^{1}$ Senior Research Engineer, Dynamic Systems \& Control Branch, Mail Stop 308, Associate Fellow.

${ }^{2}$ Professor Emeritus, Dynamic Systems \& Control Branch, Mail Stop 308, Associate Fellow.

${ }^{3}$ Senior Research Engineer, Configuration Aerodynamics Branch, Mail Stop 499, Associate Fellow.
} 


$\begin{array}{lll}\alpha & =\text { angle of attack, rad or deg } \\ \alpha_{A} & =\text { angle-of-attack amplitude, rad or deg } \\ \alpha_{0} & =\text { mean angle of attack, rad or deg } \\ \beta & =\text { sideslip angle, rad or deg } \\ \eta & =\text { state variable } \\ \theta & =\text { vector of unknown parameters } \\ \sigma^{2} & =\text { variance }\end{array}$

$\begin{array}{ll}\tau & =\text { dummy integration variable } \\ \tau_{1} & =\text { non-dimensional time constant, } \frac{1}{b_{1}}\left(\frac{V}{\ell}\right) \\ v & =\text { residuals } \\ \xi & =\text { aircraft states, }[\alpha, \beta, p, q, r]^{\mathrm{T}} \\ \phi & =\text { roll angle, rad or deg } \\ \phi_{A} & =\text { roll angle amplitude, rad or deg } \\ \omega & =\text { angular frequency, rad/sec, } 2 \pi f\end{array}$

\section{Introduction}

A erodynamicists have studied the problem of finding adequate aerodynamic models with good prediction capability since the early days of flight. Bryan's formulation [1] in 1911 established the conventional assumption that aerodynamic forces and moments depend only on instantaneous values of aircraft states and controls, which can be expressed in a linear polynomial expansion. These assumptions work well but primarily restrict the models to linear portions of the flight envelope. Adding nonlinear terms to the series expansion [2] extends the capability of the model allowing a certain class of model nonlinearity to enter the response but still no memory of previous states is captured. Improving this formulation to accommodate unsteady responses requires additional differential equations to capture the lag in response. Research efforts addressing the nonlinear unsteady problem began in the 1970's with recognition that the Volterra functional was a desirable mathematical structure [3]. This model structure, in the form of indicial models, is sufficiently general to capture a large class of nonlinear unsteady behaviors. A number of researchers have investigated this modeling problem. The modeling of unsteady aerodynamic characteristics of an aircraft from experimental data was first reported in [4]. The model equations were formulated as state-space equations. Further improvement to this modeling and parameter estimation followed in [5-7]. Many other researchers have approached the modeling problem using an indicial function formulation. In [8-9], linear indicial models were applied to data from different testing facilities and different aircraft models. Identification of indicial models from flight test data were considered in [10]. Early work addressing equations for longitudinal motion of aircraft with separate treatment of the horizontal tail can be found in [11-12] and later work placing emphasis on obtaining a model structure suitable for parameter estimation using experimental data was developed in [13]. These indicial models represented responses for the wing and tail lift separately and interference between the two lifting surfaces. In [14-15], nonlinear indicial response models were applied to the rolling 65-degree delta wing and in [16] to the prediction of a dynamically stalling wing. Nonlinear indicial models were also used in model identification from wind tunnel oscillatory data using least squares and maximum likelihood principle [17]. In [18] a method was introduced that allowed efficient identification of a linear unsteady model for an F-16XL configuration in planar forced oscillation. In this approach the aerodynamic model was formulated with static and steady-flow damping terms separate from unsteady indicial components. Enhanced System Identification (SID) and efficient testing was accomplished by using wide-band inputs. In [19] additional experiments were performed to validate the methods, mathematical models, and test techniques used in [18].

A substantial portion of the indicial modeling work described above was motivated by research for fighter aircraft configurations that continued to demand better modeling and control in an expanded flight envelope. Over approximately the last decade, supported primarily by the NASA Aviation Safety Program, unsteady modeling research at NASA Langley Research Center (LaRC) developed a range of modeling methods forming a methodology that extended the previous work. A summary of the unsteady modeling methodology is provided in this paper. The model identification problem, mathematical model structures, estimation methods, and example results are provided. This extended work considered a broader class of aircraft configurations and corresponding changes in required mathematical model structures. These changes broadened and refined both test and analysis methods. The research also reflected modeling demands for both military and civilian aircraft including a growing presence of a wide spectrum of unmanned aerial vehicles (UAV). In these cases, modeling requirements may include an expanded flight envelope for military applications or greater safety over a broader flight envelope for civilian aircraft. Unique modeling requirements also exist for both large and small UAV designs. UAV challenges stem from small UAVs that operate at very low Reynolds numbers [20], or larger complex designs, such as the NASA GL-10, with VTOL capability obtained through distributed electric propulsion (DEP) and tilt-wing features [21-23]. A common aerodynamic feature in all applications is the presence of nonlinear unsteady aerodynamic behaviors in some portions 
of the flight envelope. When these behaviors are present conventional modeling approaches are limited and more general aerodynamic models are required. More general, higher fidelity models facilitate simulation and design of predictable, controllable, and recoverable flight vehicles when subjected to adverse aerodynamic conditions.

The degree of nonlinearity and degree of unsteadiness results in one of four aerodynamic conditions that must be modeled accordingly. These four cases are (1) linear and steady (L-S), (2) linear and unsteady (L-U), (3) nonlinear and steady (NL-S), and (4) nonlinear and unsteady (NL-U). The methodology discussed in this paper addresses the approach to discerning which condition is present, which model structure is best suited to represent the physics, and how that model can be identified using SID. The first condition (L-S) is readily modeled using conventional approaches. An effective approach for the second condition (L-U) where unsteady behaviors are present, was demonstrated in [18] and [24]. In [24] a two-step regression method was applied to F-16XL forced-oscillation data. The two-step regression was first proposed in [25]. This method is advantageous when linear unsteady behaviors appear in both in-phase and out-of-phase coefficients found during harmonic analysis of forced-oscillation data. Harmonic analysis provides initial characterization of the aerodynamics by identifying where in the flight envelope unsteady or nonlinear behavior occurs, over what frequency ranges the unsteadiness is present, and it provides an estimate of the degree of aerodynamic nonlinearity. Research with the NASA Generic Transport Model (GTM) configuration found that the unsteady behavior was only present in the out-of-phase component. In that case an output error (OE) method in the time domain [26] and a nonlinear regression method in frequency domain [27] were applied to estimate model parameters. The method in [26] also made use of a "stacking" technique that combined each singlefrequency forced-oscillation response into one vector. The technique allows each frequency case to be integrated properly in the time domain but then results are combined in a single output error cost function calculation. Refs. [2627] provided further insights into modeling aerodynamic responses for configuration components of body-tail, bodywing, and body-wing-tail. Calculation of steady downwash and aerodynamic center change with respect to angle of attack were performed in [26] for the GTM configuration and in [28] for a model provided by the Central Aerohydrodynamic Institute (TsAGI). Ref. [29] included discussions on issues with identifiability and important considerations for experiment design using single-frequency forced oscillation data.

The third aerodynamic condition (NL-S) where unsteady behaviors are negligible but nonlinearities are still present has been studied extensively. In this case, stepwise regression [2] and orthogonal polynomials [30] provide a statistically rigorous way to add terms to a polynomial expansion. In [31] an advanced transport configuration, called the NASA Hybrid Wing Body, was studied in a water tunnel at Rolling Hills Research Corporation. This study used the frequency stacking technique and output error for model estimation in application to a case with nonlinear aerodynamics and negligible unsteady behaviors.

The fourth aerodynamic condition (NL-U), where the response is both nonlinear and unsteady, is more complex but manageable using a systematic approach. A systematic approach was first suggested in [32] for this more difficult problem. In that study, harmonic analysis was used to define the degree and location of nonlinear and unsteady responses. In this progressive approach, starting with careful static measurements, the model is built up using a combination of regression and output error methods. In [24, 32], it was shown how nonlinearities in the static aerodynamic measurements can contribute to higher order harmonics, thus demonstrating the importance of building up the aerodynamic models systematically and progressively from static to dynamic. A number of model structures and identification techniques were presented again in [33]. Another approach presented in [34] makes use of differential equations representing internal states that correspond to different kernels of a Volterra series. This approach includes a modeling philosophy that requires models remain compatible with classical simulation methods using look-up tables for aerodynamic data. A similar philosophy is used in NASA LaRC methods in that adding unsteady terms with clear physical interpretation and retaining conventional aerodynamic derivatives, for which a large data base and physical understanding has been built, provides a readily understood application to design and simulation.

Advances in computational power have allowed significant progress in aerodynamic modeling of nonlinear unsteady aerodynamics. Researchers have followed two paths to address the nonlinear unsteady modelling problem: a numerical path using high fidelity Computational Fluid Dynamics (CFD) techniques and an experimental path using direct measurements of aircraft responses in flight or scale models in wind tunnel dynamic tests. An approach that facilitates comparisons of wind tunnel and CFD results is to consider aircraft dynamic characteristics in the form of a general aerodynamic model identified from those results. Beyond comparisons, advances in both numerical and experimental techniques have created opportunities for the combination of these approaches to make significant improvements in handling more difficult aircraft modelling problems.

Realizing the opportunities from combining analytical and experimental approaches requires use of SID. SID provides a methodology for producing data with sufficient information content to allow model identification. Aircraft SID is an area involving a number of modeling and measurement science disciplines and the subject is well 
documented in [35]. The general steps used in aircraft SID along with modifications for unsteady modeling are discussed in Section IV. One key step is model validation where models are evaluated as predictors. The best model validation results are demonstrated by adequate prediction of aircraft responses in flight test. LaRC research in flighttest methods for real-time modeling from both subscale and full-scale air vehicles is summarized in [30, 36]. However, when flight test validation data are not available or practical to obtain then CFD predictions and ground-based experimental data are often compared to corroborate the results. Often this comparison is limited to time history comparisons. More insightful comparisons can be made by considering model parameters identified from both sets of time history data. This comparison requires estimation of parameter mean values and their uncertainty bounds in order to assess differences. Model parameters are key elements of flight dynamics simulations, stability analysis, and control design.

Although it is not common practice for wind tunnel or CFD results to be presented with associated error bounds for validation comparisons, some efforts, using various techniques, have been made to improve this shortcoming. Besides conventional methods, Design of Experiments (DOE), Statistical Process Control, and SID techniques have been applied to this problem [37-45]. Although CFD results do not contain random errors, there are potentially large uncertainties due to systematic and modeling errors. Significant uncertainty, for example, can be generated where sensitivities to turbulence models, grid selection, or time steps, are large. Application of DOE applied to computational studies is presented in [46]. Sources of uncertainty for ground-based experiments are generally known and can include a variety of systematic, modeling, and random errors such as direct measurement uncertainty and similitude errors. A body of knowledge commonly referred to as DOE [47] that addresses uncertainty problems in a statistically rigorous way provides substantial guidance for the experiment design problem. DOE has been developed over many decades and is used in many engineering and science applications. It can be used in conjunction with aircraft SID techniques and is especially effective for complex aircraft studies [21].

SID efforts also lead to specific demands on test techniques and test facility capabilities. The demand for more general, higher fidelity, models has highlighted some limitations of conventional approaches. For example, in groundbased dynamic wind-tunnel experiments where single frequency sinusoidal inputs are typically used, wide-band inputs have been shown to be more effective and less costly since fewer runs are required [18]. However, development of advanced test facilities with more automation and more advanced programmable motion capability has not been widely pursued [48]. CFD offers an opportunity to ameliorate a number of these limitations.

Initial evaluations and comparisons of stability and control (S\&C) characteristics can be made from wellestablished conventional dynamic wind-tunnel testing methods. This testing provides time histories of aerodynamic coefficients of an aircraft scale model while undergoing one-degree-of freedom forced oscillations about its body axes. Test conditions typically include selected nominal angles of attack, amplitudes, and frequency conditions. For dynamic testing, Reynolds number selection is typically limited to capabilities of the wind tunnel and dynamic test rig. Analysis of these data provides conventional in-phase and out-of-phase coefficients. Since standard errors can be estimated using the identification methodology, the analysis will facilitate more meaningful comparisons between the two sources of data. This approach suggests the importance of experiment design to allow identification of aerodynamic phenomenon and validation modeling results. In section IV examples of these types of comparisons are provided.

Because of participation by NASA in a 3-year NATO/RTO AVT-161 Task Group titled "Assessment of Stability and Control Prediction Methods for NATO Air \& Sea Vehicles," a unique opportunity was afforded to the authors to explore and validate a range of strategies for creating CFD-derived nonlinear flight simulation models within a collaborative international environment [49]. The focus of AVT-161 was to investigate the applicability of current CFD tools for predicting S\&C characteristics of air and sea vehicles. As summarized in [49] the team made remarkable progress through the leveraged efforts of many engineers and researchers from 9 countries by 1) conceiving a generic unmanned combat air vehicle (UCAV) focus configuration (Fig. 1a) called the Stability and Control Configuration (SACCON), 2) designing and building a wind-tunnel model, 3) conducting two test entries in Germany and one in the United States [40, 50], and 4) conducting and coordinating several multinational CFD studies. Experimentalists within the AVT-161 team conducted dynamic wind-tunnel tests on SACCON undergoing forced oscillation in pitch, roll, and yaw to measure the dynamic stability and aerodynamic characteristics [40, 51-52]. Other team members ran parallel dynamic computational studies following a similar approach of modeling SACCON undergoing pitch, roll, and yaw oscillation with their respective CFD tools [53-59]. The collective experiences from these studies confirmed the difficulty of achieving accurate and efficient computation of dynamic stability characteristics of highly nonlinear flows, which are highly sensitive to choice of flow solver, characteristic time steps, grid size, and turbulence models. Fortunately, a 3-year follow-on task group AVT-201 [60] pursued exploration of CFD modeling issues of the SACCON, and development of the techniques for extracting dynamic stability derivatives for flight simulation models from CFD solutions. Only a limited number of the previous AVT-161 studies addressed the reduced-order modeling 
of SACCON dynamic S\&C characteristics [56, 59, and 61]. In support of the AVT-161 effort, two studies were completed at LaRC to define best practices for CFD applied to S\&C applications. One study considered the NASA GTM transport configuration [62] and the other considered the SACCON UAV configuration [53]. These best computational practices and SID unsteady modeling methods facilitated a first comparison of high-fidelity S\&C dynamic models, including uncertainty bounds, from experimental roll forced-oscillation data and computational data simulating the same experiment for the SACCON configuration [41]. Application of both CFD and SID was again used to advantage in investigation of both low and high angles of attack aerodynamic models for the NASA GTM configuration [63]. Particular attention was given to unstable roll damping in the stall region where the sub-scale flight model presented an unfavorable wing-drop tendency. The complete aerodynamic model, estimated from dynamic wind tunnel data and computational data, was estimated with a focus on unsteady and unstable regions. In this study, data filtering, signal/noise, and bias errors were investigated to further improve the unsteady modeling methodology. A summary of CFD tool development at LaRC, addressing aircraft loss-of-control, is given in [64].

A number of techniques have been developed to improve modeling of nonlinear unsteady conditions and to take advantage of the integration of CFD, SID, and DOE technologies. In [65] the sensitivities of systematic errors on stability derivatives using a high fidelity simulation of a forced-oscillation test was done using both DOE and Monte Carlo methods. In [21] modeling of a complex aircraft with 23 factors required DOE to allow an efficient and statistically rigorous experiment to be designed. In [66] the impact of a flexible test rig, sting, and balance was investigated. This initial study was motivated by the concern that higher order models are needed to capture more severe nonlinearities and the possibility exists that aerodynamic and structural dynamic responses may be confounded in some cases. The problem can be exacerbated in the presence of systematic and random measurement errors.

This paper supports NASA's goal of improving aviation safety especially under adverse conditions such as loss of control or when nonlinear unsteady aerodynamic responses are present. Recent successful methods are presented that facilitate improved modeling fidelity for analysis, control design, and simulation. The model identification problem for conventional one-degree-of-freedom motion in wind tunnel forced-oscillation experiments is described. Mathematical model structures are presented that include conventional static and rotary dynamic terms but are extended by including indicial functions to represent unsteady responses. The goal has been to maintain the conventional stability and control derivative structure to capitalize on the large engineering knowledge base built around that structure. Model parameters describing aerodynamic properties of the system under test can be estimated using a least-squares principle. The resulting estimators form linear or nonlinear regression problems that can be formulated in the time or frequency domain. Five parameter estimation methods are described and demonstrated on experimental data from wind tunnel and water tunnel experiments. These methods are harmonic analysis, nonlinear regression, two-step linear regression, equation error, and output error. Model validation of these techniques is demonstrated with examples using SACCON, a generic UAV, Fig. 1a, two scale models of the F-16XL, a 2.5\% scale water tunnel model, Fig. 1b, and an 18\% scale wind tunnel model not shown, and the NASA Generic Transport Model (GTM), Fig. 1c.

\section{Mathematical Model Structures}

Estimation of aerodynamic parameters from response data requires postulation of a mathematical model of the aircraft dynamics. The mathematical model includes both the aircraft equations of motion and the equations for aerodynamic forces and moments, known as the aerodynamic model equations. For most of the nominal operating envelope, where the aerodynamic flows are relatively attached and steady in behavior, aerodynamic model equations are usually developed using time-invariant linear terms (stability and control derivatives). In off-nominal cases these terms often must be generalized to include time-dependent and nonlinear terms. Results from numerous studies, such as [18], have shown the dependence of aerodynamic parameters on frequency. This dependency contradicts the basic assumption that stability derivatives are time invariant. Because unsteady aerodynamics are expressed by frequency dependence, the aerodynamic model equations are effectively formulated using terms such as indicial functions or "step-response" functions to capture the unsteady response $[3,11]$.

\section{A. General Aerodynamic Model}

A general form of the aerodynamic model that allows a very broad range of indicial functions when required was suggested in [32], and earlier in [8] and [3]. Assumptions used for this general model impose only a few limitations. For this model, only rigid-body dynamics are considered along with relatively constant flight path properties such as density, viscosity, and dynamic pressure. Assuming aerodynamic coefficients are single-valued functions of aircraft states $\alpha, \beta, p, q$, and $r$, then according to Ref. [3], these coefficients can be formulated as 


$$
\begin{array}{r}
C_{a}(t)=C_{a}(0)+\int_{0}^{t} C_{a_{\xi_{1}}}(t-\tau ; \xi(\tau))^{T} \frac{d}{d \tau} \xi_{1}(\tau) d \tau \\
+\frac{\ell}{V} \int_{0}^{t} C_{a_{\xi_{2}}}(t-\tau ; \xi(\tau))^{T} \frac{d}{d \tau} \xi_{2}(\tau) d \tau
\end{array}
$$

where $C_{a}(t)$ is a coefficient of aerodynamic force or moment, $C_{a}(0)$ is the value of the coefficient at initial steady-state conditions, and $C_{a \xi}(t)$ is a vector of indicial functions whose elements are the responses in $C_{a}$ to unit steps in $\xi$. $\xi$ is defined as

$$
\xi=\left[\xi_{1} \vdots \xi_{2}\right]=\left[\begin{array}{llll}
\alpha & \beta \vdots p & q & r
\end{array}\right]^{T}
$$

and $\ell$ is the characteristic length ( $\ell=\bar{c} / 2$ or $\ell=b_{w} / 2$ ). The indicial functions approach steady-state values with increasing values of the argument $(t-\tau)$. To indicate this property, each indicial function can be expressed as

$$
C_{a_{\xi j}}(t-\tau ; \xi(\tau))=C_{a_{\xi j}}(\infty ; \xi(\tau))-F_{a_{\xi j}}(t-\tau ; \xi(\tau))
$$

where $C_{a_{\xi}}(\infty ; \xi(\tau))$ is the steady-state rate of change of the coefficient $C_{a}$ with $\xi_{j}$, while the remaining variables $\xi$ are fixed at the instantaneous values of $\xi(\tau)$, and $F_{a_{\xi j}}$ is the deficiency function. The deficiency function approaches zero as $(t-\tau) \rightarrow \infty$. When Eq. (2) is substituted into Eq. (1) the terms involving the steady-state parameters can be integrated and Eq. (1) becomes

$$
\begin{array}{r}
C_{a}(t)=C_{a}(\infty ; \xi(t))-\int_{0}^{t} F_{a_{\xi_{1}}}(t-\tau ; \xi(\tau))^{T} \frac{d}{d \tau} \xi_{1}(\tau) d \tau \\
-\frac{\ell}{V} \int_{0}^{t} F_{a_{\xi_{2}}}(t-\tau ; \xi(\tau))^{T} \frac{d}{d \tau} \xi_{2}(\tau) d \tau
\end{array}
$$

where $C_{a}(\infty ; \xi(t))$ is the total aerodynamic coefficient that would correspond to steady flow with $\xi$ fixed at the instantaneous values $\xi(t) . F_{a_{1}}$ and $F_{a_{\xi 2}}$ are vectors of deficiency functions. Further simplification of Eq. (3) is achieved by assuming that the coefficients $C_{a}$ are linearly dependent on the angular rates $\xi_{2}$. Applying a Taylor series expansion about $\xi_{2}=0$ to terms in Eq. (3) and keeping only the linear terms results in

$$
\begin{array}{r}
C_{a}(t)=C_{a}\left(\infty ; \xi_{1}(t), 0\right)+\frac{\ell}{V} \frac{\partial C_{a}}{\partial \xi_{2}}\left(\infty ; \xi_{1}(t), 0\right)^{T} \xi_{2} \\
\quad-\int_{0}^{t} F_{a_{\xi_{1}}}\left(t-\tau ; \xi_{1}(\tau), 0\right)^{T} \frac{d}{d \tau} \xi_{1}(\tau) d \tau
\end{array}
$$

or in simplified notation 


$$
\begin{array}{r}
C_{a}(t)=C_{a}(\infty ; \alpha(t), \beta(t))+\frac{\ell}{V} C_{a_{p}}(\infty ; \alpha, \beta) p(t) \\
+\frac{\ell}{V} C_{a_{q}}(\infty ; \alpha, \beta) q+\frac{\ell}{V} C_{a_{r}}(\infty ; \alpha, \beta) r(t) \\
\quad-\int_{0}^{t} F_{a_{\alpha}}(t-\tau ; \alpha(\tau), \beta(\tau)) \dot{\alpha}(\tau) d \tau \\
-\int_{0}^{t} F_{a_{\beta}}(t-\tau ; \alpha(\tau), \beta(\tau)) \dot{\beta}(\tau) d \tau
\end{array}
$$

where

$$
C_{a_{p}}=\frac{\partial C_{a}}{\partial \frac{p b}{2 V}}, C_{a_{q}}=\frac{\partial C_{a}}{\partial \frac{q \bar{c}}{2 V}} \text {, and } C_{a_{r}}=\frac{\partial C_{a}}{\partial \frac{r b}{2 V}} \text {. }
$$

The deficiency function in Eq. (4) is postulated as

$$
\begin{gathered}
F(t ; \alpha, \beta)=h(t ; \alpha, \beta) a(\alpha, \beta) \\
h(t ; \alpha, \beta)=e^{-b(\alpha, \beta) t}
\end{gathered}
$$

where $a(\alpha, \beta)$ and $b(\alpha, \beta)$ are polynomials in $(\alpha, \beta)$. When the differential operator, $D \equiv d / d t$, is introduced, then the first convolution integral in Eq. (4) can be expressed as

$$
\int_{0}^{t} F_{a_{\alpha}}(t-\tau ; \alpha(\tau), \beta(\tau)) \dot{\alpha}(\tau) d \tau=\frac{a_{1}(\alpha, \beta)}{D+b_{1}(\alpha, \beta)} D \alpha(t)=\eta_{\alpha}(t)
$$

and similarly for the second convolution integral

$$
\frac{a_{2}(\alpha, \beta)}{D+b_{2}(\alpha, \beta)} D \beta(t)=\eta_{\beta}(t)
$$

Equations (8) and (9) represent first-order linear differential equations

$$
\dot{\eta}_{\alpha}(t)+b_{1}(\alpha, \beta) \eta_{\alpha}(t)=a_{1}(\alpha, \beta) \dot{\alpha}
$$

and similarly for $\eta_{\beta}(t)$. Substituting Eq. (8) and Eq. (9) into Eq. (4) results in

$$
\begin{gathered}
C_{a}(t)=C_{a}(\infty ; \alpha, \beta)+\frac{\ell}{V} C_{a_{p}}(\infty ; \alpha, \beta) p(t) \\
+\frac{\ell}{V} C_{a_{q}}(\infty ; \alpha, \beta) q(t)+\frac{\ell}{V} C_{a_{r}}(\infty ; \alpha, \beta) r(t) \\
-\frac{a_{1}(\alpha, \beta)}{D+b_{1}(\alpha, \beta)} D \alpha(t)-\frac{a_{2}(\alpha, \beta)}{D+b_{2}(\alpha, \beta)} D \beta(t)
\end{gathered}
$$

Depending on how the aerodynamic behaviors present during testing, it is possible that some terms will reflect a nonlinear character while other terms are linear. It was often the case, for the configurations considered in this paper, that nonlinear characteristics were found primarily in the static and steady-flow dynamic components of the model, 
and only a linear form of the unsteady portion of the aerodynamic model was required. The linear aerodynamic forms for the three major terms (static, steady-flow dynamic, and unsteady) are presented in the next section.

\section{B. Linear Aerodynamic Model}

Linear aerodynamic model equations can be obtained from Eq. (11) by considering small perturbations in state variables from steady-state trim conditions. In this case the longitudinal coefficients can be expressed in terms of ( $\alpha$, $q)$ and the lateral-directional coefficients in terms of $(\beta, p, r)$. The resulting equation for the longitudinal aerodynamic coefficients is

$$
\begin{array}{r}
C_{a}=C_{a}(0)+C_{a_{\alpha}} \alpha+\frac{\ell}{V} C_{a_{q}} q \\
-\int_{0}^{t} F_{a_{\alpha}}(t-\tau) \dot{\alpha}(\tau) d \tau
\end{array}
$$

and for the lateral coefficients

$$
\begin{array}{r}
C_{a}=C_{a}(0)+C_{a_{\beta}} \beta+\frac{\ell}{V} C_{a_{p}} p+\frac{\ell}{V} C_{a_{r}} r \\
-\int_{0}^{t} F_{a_{\beta}}(t-\tau) \dot{\beta}(\tau) d \tau
\end{array}
$$

where

$$
\begin{aligned}
& F_{a_{\alpha}}=a_{1} e^{-b_{1} t} \\
& F_{a_{\beta}}=a_{2} e^{-b_{2} t}
\end{aligned}
$$

A state space form of Eq. (12a) is suggested by introducing

$$
\eta(t)=\int_{0}^{t} e^{-b_{1}(t-\tau)} \dot{\alpha}(\tau) d \tau
$$

and applying the Leibnitz integral rule, then the linear state space form is

$$
\begin{aligned}
& \dot{\eta}(t)=-b_{1} \eta(t)+\dot{\alpha}(t) \\
& C_{a}(t)=C_{a_{\alpha}}(\infty) \alpha(t)+\frac{\ell}{V} C_{a_{q}}(\infty) q(t)-a \eta(t)
\end{aligned}
$$

The four unknown parameters, $C_{a_{\alpha}}(\infty), C_{a_{q}}(\infty), a, b_{1}$, in Eq. (14) can be estimated, in principle, from measurement time histories of $C_{a}, \alpha, \dot{\alpha}$ and $q$. If these data are obtained from a one-degree-of-freedom oscillatory test in the wind tunnel (or water tunnel), then $\dot{\alpha}=q$ and the aerodynamic model equations can be expressed in the form of a transfer function

$$
\frac{C_{a}(s)}{\alpha(s)}=\frac{A s^{2}+B s+C}{s+b_{1}}
$$

where $s$ is the Laplace transform parameter and 


$$
\begin{aligned}
A & =\frac{\ell}{V} C_{a_{q}}(\infty) \\
B & =C_{a_{\alpha}}(\infty)-a+b_{1} \frac{\ell}{V} C_{a_{q}}(\infty) . \\
C & =b_{1} C_{a_{\alpha}}(\infty)
\end{aligned}
$$

A commonly used model structure that defines in-phase and out-of-phase derivatives obtained in conventional forced-oscillation experiments is suitable for the analysis of oscillatory data and obtaining initial observations of the unsteady aerodynamic character of the responses. Considering the longitudinal axis and linear aerodynamic case, the steady solution of Eq. (12a), with the nominal oscillation point $C_{a}(0)$ removed, is

$$
C_{a}(t)=\alpha_{A} \bar{C}_{a_{\alpha}} \sin (\omega t)+\alpha_{A} k \bar{C}_{a_{q}} \cos (\omega t)
$$

where $k=\pi \bar{c} f / V$ is the reduced frequency. For this conventional model form with linear aerodynamics, $C_{a}(t)$ is assumed to be functions of $\alpha, \dot{\alpha}, q$, and $\dot{q}$. The in-phase and out-of-phase components, $\bar{C}_{a_{\alpha}}$ and $\bar{C}_{a_{q}}$, can be expressed in terms of steady flow terms $C_{a_{\alpha}}(\infty), C_{a_{q}}(\infty)$ and acceleration terms or unsteady equivalent terms as

$$
\begin{aligned}
& \bar{C}_{a_{\alpha}}=C_{a_{\alpha}}(\infty)-k^{2} C_{a_{\dot{q}}} \\
& \bar{C}_{a_{q}}=C_{a_{q}}(\infty)+C_{a_{\dot{\alpha}}}
\end{aligned}
$$

In order to explain the variation of the in-phase and out-of-phase components with frequency these components were expressed in terms of deficiency functions, as in Eq. $(12 a)$. In $[8,13]$ the model for these components has the form

$$
\begin{aligned}
& \bar{C}_{a_{\alpha}}=C_{a_{\alpha}}-a \frac{\tau_{1}^{2} k^{2}}{1+\tau_{1}^{2} k^{2}} \\
& \bar{C}_{a_{q}}=C_{a_{q}}-a \frac{\tau_{1}}{1+\tau_{1}^{2} k^{2}}
\end{aligned}
$$

where $\tau_{1}=2 V / \bar{c} b_{1}$ is an aerodynamic time constant. In these aerodynamic model equations there are four unknown parameters, $a, b_{1}$ (or $\tau_{1}$ ), $C_{a_{\alpha}}$, and $C_{a_{q}}$, given in-phase and out-of-phase measurements, $\bar{C}_{a_{\alpha}}, \bar{C}_{a_{q}}$.

For one degree of freedom rolling motion in the tunnel, the lateral coefficients are assumed to be functions $\phi$ and $p$. In this case the roll angle and sideslip angle are related by the equation

$$
\beta(t)=\sin ^{-1}(\sin \alpha \sin \phi(t))
$$

The state-space form is

$$
\begin{aligned}
& \dot{\eta}(t)=-b_{1} \eta(t)+\dot{\beta}(t) \\
& C_{a}(t)=C_{a_{\beta}}(\infty) \beta(t)+\frac{b}{2 V} C_{a_{p}}(\infty) p(t)-a \eta(t)
\end{aligned}
$$

A steady oscillatory response for Eq. (12b) can be obtained as

$$
C_{a}(t)=\bar{C}_{a_{\beta}} \phi_{A} \sin (\omega t)+\bar{C}_{a_{p}} \phi_{A} k \cos (\omega t)
$$


where $\phi_{A}$ is the amplitude of roll oscillation, and $\bar{C}_{a_{\beta}}$ and $\bar{C}_{a_{p}}$ are the in-phase and out-of-phase components, respectively. These components are related to the model parameters [8] by the equations

$$
\begin{aligned}
& \bar{C}_{a_{\beta}}=C_{a_{\beta}}(\infty) \sin \alpha_{0}-a \frac{\tau_{1}^{2} k^{2}}{1+\tau_{1}^{2} k^{2}} \sin \alpha_{0} \\
& \bar{C}_{a_{p}}=C_{a_{p}}(\infty)-a \frac{\tau_{1}}{1+\tau_{1}^{2} k^{2}} \sin \alpha_{0}
\end{aligned}
$$

For one degree of freedom yaw oscillations in the tunnel, directional coefficients are assumed to be functions of $\psi$ and $r$, where yaw and sideslip angles are related by the equation

$$
\beta(t)=\sin ^{-1}(-\cos \alpha \sin \psi(t))
$$

The state space equations can be written as

$$
\begin{aligned}
& \dot{\eta}(t)=-b_{1} \eta(t)+\dot{\beta}(t) \\
& C_{a}(t)=C_{a_{\beta}}(\infty) \beta(t)+\frac{b}{2 V} C_{a_{r}}(\infty) r(t)-a \eta(t)
\end{aligned}
$$

and the corresponding in-phase and out-of-phase components are expressed as

$$
\begin{aligned}
& \bar{C}_{a_{\beta}}=C_{a_{\beta}}(\infty) \cos \alpha_{0}-a \frac{\tau_{1}^{2} k^{2}}{1+\tau_{1}^{2} k^{2}} \cos \alpha_{0} \\
& \bar{C}_{a_{r}}=C_{a_{r}}(\infty)+a \frac{\tau_{1}}{1+\tau_{1}^{2} k^{2}} \cos \alpha_{0}
\end{aligned}
$$

There are a number of parameter estimation techniques appropriate for the model structures presented here and a number of corresponding test techniques to obtain data (measured or simulated) with sufficient information content. Section IV presents the methods found effective for that purpose.

\section{Experimental and Computational Data}

A number of static and dynamic experiments were performed for several aircraft configurations in both wind and water tunnels in support of unsteady modeling research. As part of the development of computational methods, measurement predictions of several of these experiments were also made to provide simulated dynamic data for comparison and allow estimation of aerodynamic models. Examples presented use measured oscillatory data generated by different input forms and obtained at different angles of attack, amplitudes, and frequencies. From carefully designed experiments these data provide information for determining adequate aerodynamics models. The models demonstrate each of the postulated forms, presented previously, with parameters that can be estimated using the methods discussed in the next section. Both conventional and advanced modeling methods are demonstrated and used to identify aerodynamic model equations as well as in-phase and out-of-phase coefficients. Estimation of uncertainty values in wind tunnel and CFD data are limited to standard errors obtained from parameter estimation using system identification methods. For CFD simulated data, the model uncertainty will reflect uncertainty due to modeling error and turbulence model selection; uncertainty related to grid size and time steps choices are not included in this paper.

\section{Model Identification}

Different aircraft configurations can have dramatically different aerodynamic behaviors. Which part of the flight envelope is being modeled and the intended use of the models can also have a significant impact on model structure requirements. Consequently, different test techniques, estimation algorithms, and inputs are needed to identify the best mathematical model. Experiment design is the first step and a critical step in the model identification process in order 
to efficiently obtain dynamic data with sufficient information content. This applies to any test whether it is being done in flight, ground-based, or with analytical methods. Utilizing DOE methods can substantially improve the experiment design process, especially where a complex aircraft with many factors is being tested. A flow chart for aircraft system identification is represented in Fig. 2. The chart presents the general identification process followed in building a model from measured data. This process is well known and described in [35] but some aspects should be emphasized when analyzing the nonlinear unsteady problem. The choice of SID techniques and their order of application can differ for the unsteady problem. The blue arrows and boxes highlight those differences. Harmonic analysis is the first step required for the unsteady modeling problem. This step reveals under what conditions unsteady behavior is present and can indicate the degree of nonlinearity. The harmonic analysis block, in Fig. 2, lists information gained by harmonic analysis that helps resolve the model structure determination problem. In addition to using DOE methods for experiment design, specialized inputs are useful for the unsteady modeling work. The specialty inputs block presents three basic input types: single-frequency for diagnostics [32], wide-band for efficient modeling [18], and ramps for model validation [19]. Each provides unique information in the modeling process covering both estimation and validation requirements. The parameter estimation box highlights the different estimation methods needed depending on how the aerodynamics present under test conditions.

The degree of unsteady and nonlinear behaviors are commonly seen in the in-phase and out-of-phase components. Since each of the three major terms (static, steady-flow rotary dynamic, and unsteady) can be linear or nonlinear independently of the other terms, the determination of model structure for each term must be carefully addressed. Harmonic analysis is a key part of the diagnostic process that determines which of the four aerodynamic conditions (L-S, L-U, NL-S, NL-U) is present and how each of the three major terms should be characterized. The modeling philosophy represented by the model structure shown in Eqs. $(14,21$, or 25) indicates a preference to keep conventional S\&C terms for the static and steady rotary dynamic terms but adds a third term for an unsteady component when required. This approach retains the large knowledge base built up around stability and control derivatives but adds an additional term to capture any unsteady responses. The latter term is often sufficient in the form of a single-pole transfer function. Since any of these three major terms may be needed to represent linear or nonlinear aerodynamics depending on the particular aircraft, harmonic analysis is needed to systematically determine where in the envelope each term in linear or nonlinear form is required.

\section{A. Harmonic Analysis}

Ground-based dynamic tests are commonly performed as forced-oscillations about aircraft body axes at selected angles of attack, reduced frequencies, amplitudes, and Reynolds numbers. Typically, inputs are single-frequency sinusoidal variations of the Euler angles and outputs are non-dimensional aerodynamic coefficients of the forces and moments. Efficient wide-band inputs have been demonstrated [18] for this type of test and analysis. An example follows in section IV-B. A progressive approach to unsteady modeling suggested in [32] recommends, after static models have been obtained, that dynamic testing begin with single-frequency, one-degree-of-freedom, smallamplitude motions, over large range of angle of attack and sideslip. Single-frequency inputs support analysis that differentiates between nonlinear responses where higher-order harmonics are present versus a linear response where only the single-input frequency is present in the response. Using harmonic analysis these tests will reveal any nonlinear or unsteady (frequency dependent) regions in the flight envelope. The conventional linear aerodynamic model, commonly assumed in forced-oscillation experiments, is expressed as

$$
\Delta C_{a}=C_{a_{\alpha}} \alpha+\frac{\ell}{V} C_{a_{\dot{\alpha}}} \dot{\alpha}+\frac{\ell}{V} C_{a q} q+\left(\frac{\ell}{V}\right)^{2} C_{a_{\dot{q}}} \dot{q}
$$

Equation (27) is an example for the longitudinal axis but similar expressions can be written for each axis. Under harmonic inputs, this model leads to the standard expressions for the in-phase and out-of-phase coefficients.

$$
\begin{array}{ll}
\bar{C}_{a_{\alpha}}=C_{a_{\alpha}}-k^{2} C_{a_{\dot{q}}} & a=N \text { or } m \\
\bar{C}_{a_{q}}=C_{a_{q}}+C_{a_{\dot{\alpha}}} & a=N \text { or } m
\end{array}
$$

A more general mathematical model for aerodynamic coefficients, undergoing periodic motion, is 


$$
C_{a}(t)=A_{0}+\sum_{j=1}^{m} A_{j} \cos (j \omega t)+\sum_{j=1}^{m} B_{j} \sin (j \omega t)
$$

where $\omega=2 \pi f$ and $A_{0}, A_{1}, B_{1}, \ldots, A_{m}$, and $B_{m}$ are the Fourier coefficients and $C_{a}(t)$ represents one of the aerodynamic coefficients. An assumption is made that measurements are obtained as

$$
C_{a_{E}}(i)=C_{a}(i)+v(i), \quad i=1,2,3, \ldots, N
$$

where $C_{a_{E}}(i)$ are the measured values of $C_{a}(t)$ and $v(i)$ is white measurement noise with zero mean and constant variance, $\sigma^{2}$. One approach to obtain the in-phase and out-of-phase components is using Fourier transform. For the longitudinal case,

$$
\begin{aligned}
& \bar{C}_{a_{\alpha}}=\frac{2}{\alpha_{A} n_{c} T} \int_{o}^{n_{c} T} \Delta C_{a}(t) \sin \omega t d t \\
& \bar{C}_{a_{q}}=\frac{2}{\alpha_{A} k n_{C} T} \int_{o}^{n_{c} T} \Delta C_{a}(t) \cos \omega t d t
\end{aligned}
$$

where $\Delta C_{a}(t)$ is the change in response from the nominal position, $T=\frac{2 \pi}{\omega}$, and $n_{c}$ is the number of cycles of oscillation. In practice the number of cycles required must be determined from the data, however, from this body of work, 6 full cycles is often a practical minimum. Alternatively, a least squares (LS) method of harmonic analysis [24] can be applied to the time histories of measured aerodynamic coefficients to allow estimation of in-phase and out-ofphase coefficients. As follows, a LS analysis provides estimates of the Fourier coefficients, their standard errors, and the coefficient of determination, $R^{2}$. Minimizing the LS criterion

$$
J_{L S}(\theta)=\sum_{i=1}^{N}\left[C_{a_{E}}(i)-C_{a}(i)\right]^{2}
$$

where $\theta$ is the vector of unknown parameters, $\left(A_{0}, A_{1}, B_{1}, \ldots, A_{m}, B_{m}\right)$. The LS parameter estimates for Eq. (29) are given by

$$
\begin{aligned}
& \hat{A}_{0}=\frac{1}{N} \sum_{i=1}^{N} C_{a_{E}}(i) \\
& \hat{A}_{j}=\frac{2}{N} \sum_{i=1}^{N} C_{a_{E}}(i) \cos j \omega i \\
& \hat{B}_{j}=\frac{2}{N} \sum_{i=1}^{N} C_{a_{E}}(i) \sin j \omega i
\end{aligned}
$$

The corresponding parameter variances are

$$
\begin{aligned}
& s^{2}\left(\hat{A}_{0}\right)=\sigma^{2} \frac{1}{N} \\
& s^{2}\left(\hat{A}_{j}\right)=s^{2}\left(\hat{B}_{j}\right)=\sigma^{2} \frac{2}{N}
\end{aligned}
$$

for all $j$. The estimate of variance $\sigma^{2}$ is 


$$
s^{2}=\frac{1}{N} \sum_{i=1}^{N}\left[C_{a_{E}}(i)-\hat{C}_{a}(i)\right]^{2}
$$

where $\hat{C}_{a}(i)$ follows from Eq. (27) by applying final parameters estimates. The adequacy of the model given by Eq. (29) can be assessed by $R^{2}$ for each harmonic added to the model. $R^{2}$ indicates the fraction of variation in the measured data explained by the model. $R^{2}$ is expressed as

$$
R^{2}=1-S S_{E} / S_{r}, \quad 0<R^{2}<1
$$

where $S S_{E}$ is the residual sum of squares defined as

$$
S S_{E}=\sum_{i=1}^{N}\left[C_{a_{E}}(i)-\hat{C}_{a}(i)\right]^{2}
$$

and $S S_{r}$ is the total sum of squares defined as

$$
S S_{r}=\sum_{i=1}^{N}\left[C_{a_{E}}(i)-\tilde{C}_{a}(i)\right]^{2}
$$

where $\bar{C}_{a}(i)$ is the mean value.

For the pitch oscillation case, assuming $\alpha=\alpha_{A} \sin \omega t$, it follows that a model with linear aerodynamics, the inphase and out-of-phase components of $C_{a}(t)$, can be expressed in terms of the $1^{\text {st }}$ harmonic coefficients $A_{1}$ and $B_{1}$ as

$$
\begin{array}{ll}
\bar{C}_{a_{\alpha}}=\frac{B_{1}}{\alpha_{A}}=C_{a_{\alpha}}-k^{2} C_{a_{\dot{q}}} & a=N \text { or } m \\
\bar{C}_{a_{q}}=\frac{A_{1}}{k \alpha_{A}}=C_{a_{q}}+C_{a_{\dot{\alpha}}} & a=N \text { or } m
\end{array}
$$

where $k=\pi \bar{c} f / V$ is the reduced frequency. The second expression shown for each component identifies the direct relationship of the $1^{\text {st }}$ harmonic to a conventional linear aerodynamic model commonly assumed in forced-oscillation testing.

For roll oscillations, assuming $\phi=\phi_{A} \sin \omega t$ and $\beta=\phi \sin \theta$, it follows that a $1^{\text {st }}$ order harmonic model has components

$$
\begin{array}{ll}
\bar{C}_{a_{\beta}}=\frac{B_{1}}{\phi_{A}}=C_{a_{\beta}} \sin \theta-k^{2} C_{a_{\dot{p}}} & a=Y, \ell, \text { or } \mathrm{n} \\
\bar{C}_{a_{p}}=\frac{A_{1}}{k \phi_{A}}=C_{a_{p}}+C_{a_{\dot{\beta}}} \sin \theta & a=Y, \ell, \text { or } \mathrm{n}
\end{array}
$$

and for yaw oscillations, assuming $\psi=\psi_{A} \sin \omega t$ and $\beta=-\psi \cos \theta$,

$$
\begin{array}{ll}
\bar{C}_{a_{\beta}}=\frac{B_{1}}{\phi_{A}}=C_{a_{\beta}} \cos \theta+k^{2} C_{a_{\dot{r}}} & a=Y, \ell, \text { or } \mathrm{n} \\
\bar{C}_{a_{r}}=\frac{A_{1}}{k \phi_{A}}=C_{a_{r}}-C_{a_{\dot{\beta}}} \cos \theta & a=Y, \ell, \text { or } \mathrm{n}
\end{array}
$$


Examples of harmonic analysis and estimation methods are discussed together in the next section to demonstrate how harmonic analysis guides the selection of model structure and method of estimation. Examples in this section highlight the diagnostic features of harmonic analysis.

Since the first harmonic corresponds to a linear aerodynamic model and the conventional in-phase and out-ofphase components, $R^{2}$ will reflect the degree to which the linear model explains the data. As follows from Eqs. (3638), the estimates of $R^{2}$ are influenced by the value and number of Fourier coefficients (harmonic order) in Eq. (29) and the measurement noise in $C_{a_{E}}(i)$. Because the harmonic sinusoids are mutually orthogonal, the estimates of $A_{j}$ and $B_{j}$ will not change with the number of model terms included in Eq. (29). Changes will only appear in the corresponding standard errors and residuals defined by Eq. (37). This makes $R^{2}$ an effective diagnostic tool to discern the adequacy of a linear first-order model against nonlinear higher harmonic models.

If the static and steady-flow damping terms are assumed to be nonlinear and well represented by higher order polynomials, then the order determined by harmonic analysis also implies the order of the polynomial required. For example, consider a conventional harmonic model for rolling moment analysis

$$
\Delta C_{l}=C_{l_{\beta}} \beta+\frac{\ell}{V} C_{l \dot{\beta}} \dot{\beta}+\frac{\ell}{V} C_{l p} p+\left(\frac{\ell}{V}\right)^{2} C_{l \dot{p}} \dot{p}
$$

In this case, assuming $\phi=\phi_{A} \sin \omega t$ and $\beta=\phi \sin \theta$, it follows that a $1^{\text {st }}$ order harmonic model is

$$
\Delta C_{l}=\phi_{A}\left(C_{l_{\beta}} \sin \theta-k^{2} C_{l \dot{p}}\right) \sin \omega t+\phi_{A} k\left(C_{l p}+C_{l \dot{\beta}} \sin \theta\right) \cos \omega t
$$

If a cubic nonlinearity exists in the static term, such that Eq. (21) contains the term $C_{\ell \beta^{3}} \beta^{3}$, then this term produces a cubic-harmonic sine term. Using the identity, $\sin ^{3} \omega t=1 / 4[3 \sin \omega t-\sin 3 \omega t]$, it can be shown that both $1^{\text {st }}$ and $3^{\text {rd }}$ harmonic terms are added to the model to produce

$$
\begin{gathered}
\Delta C_{l}=\phi_{A}\left(C_{l_{\beta}} \sin \theta-k^{2} C_{l \dot{p}}+(3 / 4) \phi_{A}^{2} C_{\beta^{3}} \sin ^{3} \theta\right) \sin \omega t \\
+\phi_{A} k\left(C_{l p}+C_{l \dot{\beta}} \sin \theta\right) \cos \omega t \\
-(1 / 4) \phi_{A}^{3} C_{\beta^{3}} \sin ^{3} \theta \sin 3 \omega t
\end{gathered}
$$

Eq. (44) shows that the cubic nonlinearity expresses itself as both a $3^{\text {rd }}$ order harmonic and an additional component added to the $1^{\text {st }}$ harmonic term. A similar analysis can be done for harmonics of different order and the same analysis can be applied to the steady-flow damping term. Thus, the presence of a $3^{\text {rd }}$ order term in the static data or in the steady-flow damping term will result in a $3^{\text {rd }}$ harmonic during a general harmonic analysis. Unfortunately, the unsteady term does not lend itself to this type of analysis. In general independent information about each of the three major model terms is required to determine the source of higher harmonic response. Fortunately, the three major terms can be independently characterized: steady flow (static) terms can be measured independently in a static test; the two major dynamic terms are measured together in dynamic tests however one represents steady flow (frequency independent) and the other represents unsteady flow (frequency dependent). Details of this more general analysis are provided in [32].

Applying harmonic analysis to roll oscillation data for the NASA GTM produces the graphic shown in Fig. 3. Without detriment to this discussion, ordinate values are removed on all GTM plots in order to maintain proprietary agreements. Analysis was performed on data pre-filtered at $4 \mathrm{~Hz}$. The filter was applied in both directions to ensure no lags were introduced into the response data. Filtering issues are addressed in detail for this case in [63], however, it was found that the in-phase and out-of-phase components were relatively insensitive to filter cutoff frequencies above $2 \mathrm{~Hz}$. In this figure the in-phase and out-of-phase components and the coefficient of determination are plotted against angle of attack for five frequencies and one selected roll-oscillation amplitude. This experiment was conducted in the NASA Langley 14x22 Wind Tunnel. Model geometry is given in Fig. 1c. This type of plot highlights the location of unsteady behaviors. The plot shows little frequency dependence (unsteady behavior) for the in-phase component 
(effective dihedral, $\bar{C}_{l_{\beta}}$ ) while the out-of-phase component (damping term, $\bar{C}_{l_{p}}$ ) presents unsteady behaviors at $\alpha_{0}$ $=12^{\circ}$ and for $24^{\circ} \leq \alpha_{0} \leq 30^{\circ}$. Some unsteady behavior is also present for $\alpha_{0}>40^{\circ}$. Roll damping instabilities are present at $\alpha_{0}$ near $12^{\circ}, 14^{\circ}$, and above $40^{\circ}$. $R^{2}$ shows substantially low values in the stalled flight region for $12^{\circ} \leq \alpha_{0}$ $\leq 40^{\circ}$. This implies that either a linear model is not satisfactory or significant noise levels are present. Final determination of model nonlinearity is accomplished by considering measurement and harmonic model time histories.

To demonstrate the diagnostic process, time histories of response variables versus input variables are shown in Fig. 4 for the three lowest $R^{2}$ cases at $\alpha_{0}=\left(14^{\circ}, 22^{\circ}, 30^{\circ}\right)$ and for low and high frequencies. For these types of plots a regular ellipse corresponds to a linear aerodynamic response [67]. In order to highlight ellipse shapes the scales are not the same. The left side presents plots for the low frequency cases at $f=0.06 \mathrm{~Hz}$, and the right side shows high frequency cases at $f=0.92 \mathrm{~Hz}$.

The first case at $\alpha_{0}=14^{\circ}$, demonstrates a nonlinear response with steady dynamics (NL-S). In this case the very low $R^{2}$ value for the high frequency data (Fig. 3) correctly indicated a nonlinear response since the high frequency plot for $C_{l}$ is an irregular ellipse (Fig. 4a), and linear aerodynamics can only produce regular ellipses. The linear response from the $1^{\text {st }}$ harmonic model is shown as a green ellipse and the $3^{\text {rd }}$ order harmonic, nonlinear response, as a red ellipse. Nonlinear responses are often expected in the lateral-directional axes where a strong cubic nonlinearity typically exists in the static terms with respect to sideslip. This case is also a good example of where engineering judgment is required. Although the response at high frequency is nonlinear, respect for the very small values of the derivatives may lead to a conclusion that the added complexity of a nonlinear model is not warranted. $R^{2}$ does not account for overall magnitude of the response variables.

The second case at $\alpha_{0}=22^{\circ}$, demonstrates a linear response with steady dynamics (L-S). In this case the low $R^{2}$ value occurred at the lowest frequency (Fig.3). In this case higher noise levels reduced $R^{2}$. Plots in Fig. $4 \mathrm{~b}$ show that linear models match the measured data reasonably well except for the low frequency case of the rolling moment, where the response measurement has substantial noise in spite of filtering and averaging the responses over numerous cycles. In this case, although the noise has reduced the $R^{2}$ metric, the estimated linear model is still an adequate representation of the response.

The third case at $\alpha_{0}=30^{\circ}$, presents a linear response with unsteady dynamics (L-U). In this case, where an unsteady model is required, the low $R^{2}$ value occurred at the lowest frequency (Fig. 3). Plots in Fig. 4c show linear models match the measured data reasonably well except for the low frequency case of the rolling moment where the response measurement has substantial noise. Similar to the last case, although noise has reduced the value of $R^{2}$, the estimated linear-unsteady model is still a reasonable representation of the response.

For the set of amplitudes and frequencies considered in this example using the NASA GTM, the fourth modeling case NL-U was not observed for the lateral axis; however, it did present in the directional axis. Details of the NL-U case for GTM was presented in [27], where a third order harmonic was required to model the cubic response in yawing moment to oscillations in sideslip. The source of the nonlinearity was found to be in the static term because of a strong cubic anti-symmetric relationship of yawing moment to sideslip. This type of static nonlinearity is common in the lateral-directional axes. A more general example of the NL-U case, with nonlinear models in all three major terms, is presented in Section IV.B using F-16XL data.

\section{B. Estimation Methods}

A number of approaches are available for estimating parameters in aerodynamic model equations. Methods, such as U.S. Air Force DATCOM, are based on extensive rules for aerodynamic dependencies and aircraft geometry. This approach is limited to low angle of attack and low rotational rate cases. Analytical methods, such as CFD where general physics-based equations define the mathematical model, are applicable to the full aircraft flight envelope. However, CFD applied to S\&C problems is still an area of research [60]. Although these methods work well to varying degrees, the best aerodynamic predictions are still obtained using experimental methods. Full-scale flight experiments provide the most direct measurements of aerodynamic behaviors but this is the most expensive approach and not available in early design phases. The wind tunnel provides the next best approach with current technology; however, this approach has a number of difficulties such as scaling and tunnel effects. Another difficulty for tunnel testing is that often certain dynamic tests are not possible or easily produced in tunnels with conventional test rigs.

An opportunity to improve modeling technology combines both wind tunnel and CFD approaches. In this paper the aerodynamic parameters are estimated primarily from wind tunnel dynamic tests to demonstrate modeling methodology. However, model estimation results from CFD simulations are also provided to highlight comparisons and demonstrate some of the successes using CFD applied to S\&C problems. 


\section{Nonlinear Regression}

This example highlights the L-U case with frequency dependence present only in the out-of-phase component. Harmonic analysis performed on pitch oscillatory data is shown in Fig. 5 for the NASA Generic Transport Model (GTM). Ordinate values are removed to maintain proprietary agreements for this model. For this configuration the inphase component, $\bar{C}_{N_{\alpha}}$, does not vary with frequency but without an unsteady component, its value agrees closely with $C_{N_{\alpha}}$ from steady measurements. On the other hand the out-of-phase component, $\bar{C}_{N_{q}}$, shows a strong variation with frequency and presents an unsteady region for $f<0.43 \mathrm{~Hz}$ and $10^{\circ}<\alpha_{0}<50^{\circ}$. Values of $R^{2}$ are very good over the full range of angle of attack but indicate limited degradation of the linear model for $\alpha_{0}>35^{\circ}$. Given this set of circumstances and frequency dependent measurements, a suitable approach for estimating the three unknown parameters, $a, \tau_{1}$, and $C_{a_{q}}$, given in Eq. (19), is to use a nonlinear regression method [27]. For this case a general model is formed as

$$
z(j)=g[x(j), \theta]+v(j), \quad j=1,2, \ldots, m
$$

where $x(j)$ is a vector of regressors computed from measured data at the jth data point, $g$ is a nonlinear function of $x(j)$, and unknown parameters are given by the vector, $\theta$. The least-squares estimator can be obtained by minimizing the sum of squared errors

$$
J_{N R}(\theta)=\sum_{j=1}^{m}\{z(j)-g[x(j), \theta]\}^{2}
$$

As a check on regression fit, parameter estimates are applied to Eq. (19) and used to generate the plot in Fig. 6. The figure shows a good fit between measured and estimated values of $\bar{C}_{N_{q}}$ at $\alpha_{0}=18^{\circ}$. As a check on model prediction,

Fig. 7 shows the variation of $C_{N}$ with $\alpha$ at $f=0.86 \mathrm{~Hz}, \alpha_{A}=10^{\circ}$, and two nominal values of $\alpha_{0}=\left(14^{\circ}, 26^{\circ}\right)$. In both cases the shape of the predicted data forms a regular ellipse reflecting the underlying linear aerodynamic model structure. The measured $C_{N}$ shows some deviation from linear behavior (irregular ellipse), in the pre-stall and stall regions.

2. Two-Step Linear Regression

This regression method [24] can be applied in L-U cases where both the in-phase and out-of-phase components present frequency dependence and a linear aerodynamic model is adequate. An aerodynamic model of an aircraft performing a one degree-of-freedom oscillatory motion about one of its body axes can be formulated in terms of the in-phase and out-of-phase components for rolling motion as in Eq. (23) or in a compact form as

$$
\begin{gathered}
\bar{C}_{a_{\beta}}=C_{a_{\beta}}(\infty) \sin \alpha-a f_{1} \sin \alpha \\
\bar{C}_{a_{p}}=C_{a_{p}}(\infty)-a f_{0} \sin \alpha
\end{gathered}
$$

where

$$
\begin{aligned}
& f_{1}=\frac{\tau_{1}^{2} k^{2}}{1+\tau^{2} k^{2}} \\
& f_{0}=\frac{\tau_{1}}{1+\tau_{1}^{2} k^{2}}
\end{aligned}
$$

and subscript $a$ denotes appropriate force or moment coefficient. The corresponding equations for pitching motion are given in Eq. (19) or as 


$$
\begin{aligned}
& \bar{C}_{a_{\alpha}}=C_{a_{\alpha}}(\infty)-a f_{1} \\
& \bar{C}_{a_{q}}=C_{a_{q}}(\infty)-a f_{0}
\end{aligned}
$$

and for yawing motion in Eq. (26) or as

$$
\begin{aligned}
& \bar{C}_{a_{\beta}}=C_{a_{\beta}}(\infty) \cos (\alpha)-a f_{1} \cos (\alpha) \\
& \bar{C}_{a_{r}}=C_{a_{r}}(\infty)+a f_{0} \cos \alpha
\end{aligned}
$$

With the expression,

$$
\frac{\tau_{1}^{2} k^{2}}{1+\tau_{1}^{2} k^{2}}=1-\frac{1}{1+\tau_{1}^{2} k^{2}}
$$

Considering rolling motion, Eq. (47) can be rearranged into a set of equations with $m$ different values of $\mathrm{k}$ as

$$
y(j)=a_{0}+a_{1} x(j), \quad j=1,2, \ldots, m
$$

where

$$
\begin{aligned}
& x=\bar{C}_{a_{\beta}}, y=\bar{C}_{a_{p}} \\
& a_{0}=C_{a_{p}}(\infty)+a_{1}\left(a-C_{a_{\beta}}(\infty)\right) \sin \alpha \\
& a_{1}=-\tau_{1}
\end{aligned}
$$

In the first step a linear regression is used in estimation of parameters $a_{0}$ and $a_{1}$ in Eq. (52) from measured in-phase and out-of-phase components at $m$ different frequencies, $m>2$.

The second step of regression follows from Eqs. (47-48) replacing $\tau_{1}$ by its estimated value. The resulting equations are

$$
\begin{aligned}
& y_{1}(j)=d_{0}+d_{1} x_{1}(j), \quad j=1,2, \ldots, m \\
& y_{2}(j)=c_{0}+d_{1} x_{2}(j), \quad j=1,2, \ldots, m
\end{aligned}
$$

where for rolling oscillations these terms are

$$
\begin{aligned}
y_{1}(j)=\bar{C}_{a_{\beta}}(j), \quad y_{2}(j)=\bar{C}_{a_{p}}(j) \\
x_{1}(j)=-f_{1}(j) \sin \alpha, \quad x_{2}(j)=-f_{0}(j) \sin \alpha \\
d_{0}=C_{a_{\beta}}(\infty) \sin \alpha, \quad d_{1}=a \sin \alpha, \quad c_{0}=C_{a_{p}}(\infty)
\end{aligned}
$$

Similar expressions can be written for pitching and yawing oscillations. Development of regression equations and estimator properties can be found in [35].

Application of the two-step regression method to roll oscillatory data was demonstrated for an $18 \%$ scale F-16XL aircraft (2.5\% scale model shown in Fig. 1b) in the NASA Langley 14x22 Wind Tunnel. Harmonic analysis, shown in Fig. 8, indicates unsteady behavior (frequency dependence) for $25^{\circ} \leq \alpha_{0} \leq 45^{\circ}$ and $R^{2}$ confirms the predominantly linear behavior with the exception of $\alpha_{0}=45^{\circ}$. Linear dependence of in-phase and out-of-phase components, defined by Eq. (52), is confirmed in Fig. 9. The slope of these data define time constant, $\tau_{1}$. Variation of the parameter estimates 
and $2 \sigma$ error bounds with $\alpha$ are shown in Fig. 10. A comparison of measured and predicted rolling moment coefficient is shown in Fig. 11.

Demonstrating the utility of CFD simulated dynamic data and SID in application to forced-oscillation experiments was first accomplished in [41]. In this case the two-step regression method was applied to roll oscillatory data from tests reported in [40] for the SACCON aircraft (Fig. 1a). Harmonic analysis, Fig. 12, shows the L-U case was found at $\alpha=20^{\circ}$ and L-S for the other test points. Application of the two-step regression method to wind tunnel data produced parameter estimates and their standard errors, Table I.

Table I. Two-step Regression estimates for unsteady model at $\alpha_{0}=20^{\circ}$ and $\alpha_{A}=5^{\circ}$, SACCON.

\begin{tabular}{|c|c|c|c|c|c|}
\hline & \multicolumn{2}{|c|}{ Step 1 } & \multicolumn{3}{c|}{ Step 2 } \\
\hline & $b_{1}$ & $\tau_{1}$ & $a$ & $C_{l p}$ & $C_{l \beta}$ \\
\hline$\hat{\theta}$ & 3.68 & 6.37 & 0.75 & -0.40 & 0.57 \\
\hline$\hat{\sigma}(\theta)$ & $(0.34)$ & $(0.58)$ & $(0.04)$ & $(0.047)$ & $(0.05)$ \\
\hline
\end{tabular}

Consistency and fit of the linear relationship in Eq. (52) is shown in Fig. 13 for the wind tunnel data. A similar check of consistency for the CFD simulated data is shown in Figs. 14-15. As part of the investigation, two different turbulence models were evaluated. Two-step regression of the CFD simulated data using the SA turbulence model produced Fig. 14 and using the SST turbulence model produced Fig. 15. In these results the SA model appeared to support the linear relationship except for the lowest frequency point. The study in [41] did not investigate the low frequency point to determine if it was an outlier, a deficiency in the CFD approach at low frequency, or if it reflected an aerodynamic characteristic not captured by the current model structure. Instead the L-U model structure was retained to realize the benefits of a parsimonious regression model and to allow direct comparisons of models. The impact of that assumption was larger uncertainty bounds on the estimated parameters. Results for the SST case show the same characteristic for the low frequency data point and presents a more quadratic shape with frequency.

A direct comparison of the four model parameter estimates and their $2 \sigma$ standard deviations is shown in Fig. 16 for wind tunnel, SA, and SST cases. For the wind tunnel case, uncertainty in the model parameters is derived from measurement noise and model error. For the CFD cases, uncertainty is derived only from two sources of model error. The first source of model error is due to differences in the L-U model structure representations of SA or SST data. This error is reflected in the $2 \sigma$ bounds for each case. The second source of model error results from the two turbulence model representations of the data. The two turbulence models represent a range of model choice that is part of the model uncertainty. The charts for $C_{l_{p}}$ and $C_{l_{\beta}}$, in Fig. 16, show the uncertainty bars overlapping for all three cases. The implication is that no statistical difference exists between cases for these parameters, at the 95\% confidence level. This result is for a single-case study; consequently the results are not under statistical process control [39]. A Monte Carlo approach was not an option for this study due to the computational burden. Another option with less computational burden is to use DOE or a Gaussian Process method to add statistical rigor to the results. However, even without adding those options, it is informative to account for any uncertainty within a single case study as demonstrated in Fig. 16. The two charts on the right side of Fig. 16 show the parameters for the unsteady component of the model. The time constant $\tau_{1}$ for the wind tunnel and SA cases are in statistical agreement but the SST case produced a statistically different result. For the attenuation factor, $a$, the SA and SST are in statistical agreement but not with the wind tunnel case.

Time histories of measured and predicted rolling moment responses for the highest and lowest frequencies are shown in Fig. 17 as a validation check. The SA model is in very good agreement with the wind tunnel model and provides a good fit to the measured data. The SST model shows some difference with the both SA and wind tunnel measurements. In spite of steady damping terms from wind tunnel and SST models being in reasonable agreement the differences in the unsteady components are sufficient to show response prediction differences. Differences between SA and SST model parameters and response predictions reflect the range of uncertainty present due to selection of turbulence models.

3. Equation Error Method

In some cases, especially if wide-band inputs are used, the L-U problem is addressed more efficiently using frequency domain model structures. The Equation Error (EE) Method is a linear regression method that can be developed either in the time domain or the frequency domain. However, considering the state-space representation in Eq. (14), the time domain approach is not directly possible because $\eta$ and $\dot{\eta}$ are not measureable variables. For the 
frequency domain approach the model is given by transforming Eq. (14) into transfer function form and expressing $s$ as $i \omega$. In this case the model and output equation have the form

$$
\begin{aligned}
& C_{N}(\omega)=\frac{-A \omega^{2}+C+B i \omega}{b_{1}+i \omega} \alpha(\omega) \\
& z(j)=C_{N}(j)+v(j), \quad j=1,2, \ldots, m
\end{aligned}
$$

where $C_{N}(\omega)$ and $\alpha(\omega)$ are the Fourier transforms of $C_{N}(t)$ and $\alpha(t) . z(j)$ are the measurements, $v(j)$ is measurement noise, $m$ is the number of frequencies at which the transformed input output data are known, $\omega$ is the angular frequency, and $i=\sqrt{-1}$. In the Equation Error formulation unknown parameters are estimated from minimization of

$$
J_{E E}(\theta)=\sum_{j=1}^{m}\left|C_{N}(j)\left(b_{1}+i \omega_{j}\right)+\left(A \omega_{j}^{2}-C-B i \omega_{j}\right) \alpha(j)\right|^{2}, \quad j=1,2, \ldots, m
$$

In this formulation $v(j)$ are residuals that encompass measurement noise and equation errors. Parameters $A, B$, and $C$ are related to the aerodynamic coefficients by Eq. (16).

An example of this method was applied to measurements obtained from pitch oscillation tests of a $2.5 \%$ scale F16XL model in a water tunnel [18]. As an efficient alternative to conventional single-frequency forced-oscillation test inputs, Schroeder sweeps were introduced. This approach was found to provide substantially more efficient and more effective input for identification of unsteady models and was validated in [19]. Practical identification using a singlefrequency input would suggest at least 6 runs, each at a different frequency, to ensure the four parameter model is adequately identified. For this example, a Schroeder sweep was used to allow model identification in one forcedoscillation run at $\alpha_{0}=42.5^{\circ}$. Figure 18 shows the input, $\alpha(t)$, and output, $C_{N}(t)$, displacements from their starting values at $\alpha_{0}=42.5^{\circ}$. The power spectrum of the Schroeder sweep is shown in Fig. 19. Schroeder sweeps provide a flat power spectrum over a specified frequency band with low peak-to-peak amplitudes. Amplitude of the Schroeder sweep is controlled by properly phasing each harmonic. Harmonic analysis provided the in-phase and out-of-phase components shown in Fig. 20. In this case, both components present strong frequency dependence for $30^{\circ} \leq \alpha \leq 70^{\circ}$, indicating an unsteady model is needed for that angle of attack region. Final estimates from the EE approach are shown with the Output Error (OE) results in the next section. In this study the EE estimates were used as initial estimates or starting values for the optimization process inherent in the OE method.

4. Output Error Method (Frequency Domain)

The output error (OE) method in the frequency domain can be applied directly using Eq. (56) to address this form of the L-U problem. OE methods are convenient when the estimation problem is nonlinear. However, the linearity of the aerodynamic model is not changed by the form of the estimation problem. In this case parameter estimates for the linear aerodynamic model are obtained by minimization of the mean square output error.

$$
\begin{aligned}
& J_{O E}(\theta)=\sum_{j=1}^{m} \tilde{v}^{*}(j) \tilde{v}(j) \\
& \tilde{v}(j)=\tilde{z}(j)-\frac{-A \omega^{2}(j)+C+B i \omega(j)}{b_{1}+i \omega(j)} \tilde{\alpha}(j)
\end{aligned}
$$

The OE approach was applied to the same wide-band forced-oscillation data as the EE method discussed previously. Figure 21 shows the parameter estimates and their standard errors over the range of angle of attack where unsteady behaviors were observed. These estimates are consistent with the values obtained from the EE method. Estimate from the two methods in the frequency domain (f.d.) are compared in Table II for $\alpha_{0}=42.5^{\circ}$. Additional OE time domain (t.d.) results are also shown for comparison.

The two methods show very good agreement for the parameter estimates and the output error method obtained slightly better standard errors. Validation of these estimates is demonstrated by application to test data not used for 
estimation. Fig. 22 shows the output error estimates applied to ramp-and-hold data for a model validation test. The time histories show a very good match.

Table II. Parameter estimates for unsteady model at $\alpha_{0}=42.5^{\circ}, \mathrm{F}-16 \mathrm{XL}, 2.5 \%$ scale.

\begin{tabular}{|c|c|c|c|c|c|c|}
\hline & \multicolumn{2}{|c|}{ Equation Error (f.d.) } & \multicolumn{2}{|c|}{ Output Error (f.d.) } & \multicolumn{2}{|c|}{ Output Error (t.d.) } \\
\hline$\theta$ & $\hat{\theta}$ & $s(\hat{\theta})$ & $\hat{\theta}$ & $s(\hat{\theta})$ & $\hat{\theta}$ & $s(\hat{\theta})$ \\
\hline $\mathrm{A}$ & 0.939 & 0.063 & 0.895 & 0.082 & \multicolumn{2}{|c}{} \\
\hline $\mathrm{B}$ & 2.571 & 0.055 & 2.521 & 0.064 & \multicolumn{2}{|c|}{ not applicable } \\
\hline $\mathrm{C}$ & -0.090 & 0.051 & -0.101 & 0.019 & \multicolumn{2}{|c|}{} \\
\hline$b_{1}$ & 0.138 & 0.026 & 0.144 & 0.013 & & 0.025 \\
\hline$C_{N \alpha}$ & -0.65 & 0.39 & -0.70 & 0.15 & -0.35 & 0.045 \\
\hline$C_{N q}$ & 2.79 & 0.19 & 2.66 & 0.24 & 2.60 & 0.025 \\
\hline$a$ & -3.09 & 0.39 & -3.09 & 0.16 & -2.80 & 0.29 \\
\hline$\tau_{1}$ & 21.53 & 4.11 & 20.57 & 1.90 & 17.48 & \\
\hline
\end{tabular}

\section{Output Error Method (Time Domain)}

The estimation methods presented so far have been applied to linear aerodynamic models (L-S, L-U). These methods can be used to estimate all parameters in the three major model terms, however, an OE estimation method is needed when more general nonlinear aerodynamic model structures (NL-S, NL-U) are required. In [32], a general mathematical model structure and modelling methodology was presented to address the nonlinear unsteady case. The methodology suggests using the least complex model that provides an adequate representation of the aerodynamic response. With this approach, a progression is made toward more complex models and correspondingly more complex experiment design. For example, modeling and removing static nonlinear responses first can often lead to simplified estimation requiring only linear model structures for the remaining two major terms. The simplifying assumptions associated with the least complex model are removed in progression, only as required to achieve model adequacy. In general, this approach should lead to the best predictive model.

In this section two examples are provided. The first is to highlight a general NL-U case where all three major aerodynamic terms are nonlinear. The second is to highlight application of CFD to an S\&C problem where insights into the aerodynamic responses and modeling requirements were provided by the computational modeling approach.

The first example using measurements from a single-axis forced-oscillation experiment in roll with a $2.5 \%$ scale F-16XL model is presented here to demonstrate the more general method [32]. Tests were performed in a water tunnel at the Rolling Hills Research Corporation. An additional example of the NL-U case using a transport model during single-axis yaw oscillations is available in [27] where nonlinearities were required for the static terms only. For the F16XL example, measurements were obtained at six non-dimensional frequencies, $k=[0.066,0.095,0.131,0.160$, $0.197,0.262]$, for a range of angle of attack, during large amplitude $\left(\phi_{A}=30^{\circ}\right)$ roll oscillation tests. Harmonic analysis is presented in Fig. 23 where frequency dependent behavior is observed for $25^{\circ} \leq \alpha \leq 40^{\circ}$. The middle plot presents $R^{2}$ for a first harmonic model representing the linear case. The linear models do not completely explain the total variation present in the data at the lower frequencies. The lower plot presents $R^{2}$ for $1^{\text {st }}, 2^{\text {nd }}$, and $3^{\text {rd }}$ order harmonic models. A dramatic improvement in $R^{2}$ occurs only when a $3^{\text {rd }}$ order harmonic model is used. For the $k=0.066$ case, $R^{2}$ changes from 0.78 , with the $1^{\text {st }}$ harmonic model, to 0.97 when a $3^{\text {rd }}$ order harmonic model is used. One contribution to the nonlinear behavior is from the static terms in the model. Fig. 24 shows the fairly severe nonlinear character of the roll moment over angle of attack and sideslip. The surface is relatively smooth and linear at low and very high angles of attack; however in the mid angle-of-attack range, $20^{\circ} \leq \alpha \leq 50^{\circ}$, fairly severe static nonlinearities occur.

For a single-degree-of-freedom roll test it is reasonable to assume that $C_{l}$ can be expressed as $C_{l}(\beta, p)$ and that a slightly more general model structure, given in Eq. (11), is appropriate. Angle of attack can be considered fixed at a nominal value because only relatively small changes occur during single degree-of-freedom roll forced oscillations with $\phi_{A} \leq 20^{\circ}$. The model for this case can be written in state form as 


$$
\begin{aligned}
& \dot{\eta}(t)=-b_{1}(\beta) \eta(t)-a(\beta) \dot{\beta} \\
& C_{a}(t)=C_{a}(\infty ; \beta)+\frac{l}{V} C_{a_{p}}(\infty ; \beta) p(t)+\eta(t)
\end{aligned}
$$

A broad class of nonlinear unsteady responses can be modelled with this basic model structure where the static term, steady-flow damping term, and indicial term parameters can vary nonlinearly with $\beta$. In this example, it is assumed that each of the four unknown parameters $\left(C_{l}(\infty ; \beta), C_{l p}(\infty ; \beta), a(\beta), b_{1}(\beta)\right)$ can be represented by polynomials in $\beta$. In general, experiments can be designed to identify models for the static, damping, and indicial terms separately, although few facilities are capable of executing the motions required. Some computational methods are being investigated to obtain indicial models where specialized motions can be applied more readily [68]. For this example, using the $\mathrm{OE}$ formulation in the time domain, unknown parameters are estimated through minimization of $J_{O E}$.

$$
\begin{aligned}
& J_{O E}(\theta)=\sum_{i=1}^{N} v^{2}(i) \\
& v(i)=C_{a}(i)-C_{a}(\infty ; \beta(i))-\frac{l}{V} C_{a_{p}}(\infty ; \beta(i)) p(i)-\eta(i)
\end{aligned}
$$

where $\eta(\mathrm{i})$ is computed from the state equation, Eq. (56). In this example only conventional single-frequency, singleaxis, forced-oscillation test data is used. Figure 25 shows sample time histories of the large amplitude oscillatory data at $\alpha_{0}=37.5^{\circ}$ and $\beta_{0}=0^{\circ}$. The figure shows four cycles of oscillation for four frequencies, $k=[0.066,0.095,0.131$, 0.160]. Time-domain data at each frequency for the input and output measurements are stacked to ensure all frequency content is included in the analysis. Integration of the model equations must be performed respecting the different conditions at each frequency. The first cycle of predicted response is eliminated to remove any initial transients and ensure that steady harmonic motion is used in calculating residuals. The amplitude, $\phi_{A}$, is the same for each frequency; however, the resulting $\alpha$ and $\beta$ oscillations change during roll oscillations according to the kinematic relationships:

$$
\begin{aligned}
& \beta=\sin ^{-1}\left\{\sin (\phi) \sin \left(\theta_{0}\right) \cos \left(\psi_{0}\right)-\cos (\phi) \sin \left(\psi_{0}\right)\right\} \\
& \alpha=\tan ^{-1}\left\{\cos (\phi) \tan \left(\theta_{0}\right)+\frac{\sin (\phi) \sin \left(\psi_{0}\right)}{\cos \left(\theta_{0}\right) \cos \left(\psi_{0}\right)}\right\}
\end{aligned}
$$

where $\theta_{0}$ and $\psi_{0}$ are the nominal pitch and yaw offsets, respectively. The distortion of the rolling moment response from a steady harmonic wave, in particular for the low frequency cases, indicates nonlinear behavior.

Under the constraint of one-degree-of-freedom harmonic data, an iterative 2-stage process shown in Fig. 26 can be used to identify the complete model. Before starting the process, initial parameter values for a linear model are estimated using one of the methods described previously. Small amplitude motions tend to be well represented by linear models. Polynomials of the static values should be well modelled since these values can be directly measured in the wind tunnel; however for this example, the static terms were estimated from the dynamic measurements.

To start the 2-stage process, first model structure determination of the unsteady term polynomials, $a(\beta)$ and $b(\beta)$, is performed using stepwise regression (SR) described in [35]. SR is an extension of linear regression that includes identification of statistically significant mathematical model structures. To setup the regression equation, a variable $y(t)$ is formed by subtracting the static term and approximated steady-flow damping term from the force or moment measurement, $C_{a}(t)$, as

$$
y(t)=C_{a}(t)-C_{a}(\infty ; \beta)-\frac{\ell}{V} C_{a_{p}}(\infty ; \beta) p(t)
$$

Then the regression equation, showing the discrete measured values at time $t(i)$, is given as

$$
\dot{y}_{E}(i)=-\left[b_{1}\left(\beta_{E}(i)\right) y_{E}(i)+a\left(\beta_{E}(i)\right) \dot{\beta}_{E}(i)\right]+\varepsilon_{\eta}(i), \quad i=1,2, \ldots, N
$$


where index $E$ indicates the measured values and $\varepsilon_{\eta}(i)$ is an equation error. Derivatives of measurement, $y_{E}$, are formed using a locally smoothed numerical derivative [35]. Parameter estimates are then updated with output error estimation. State and measurement equations for OE estimation are

$$
\begin{aligned}
& \dot{\eta}(t)=-b_{1}(\beta) \eta(t)-a(\beta) \dot{\beta}_{E} ; \eta(t=0)=\eta(0) \\
& y_{E}(t)=C_{a_{E}}(i)-C_{a}\left(\infty ; \beta_{E}(i)\right)-\frac{\ell}{V} C_{a_{p}}\left(\infty ; \beta_{E}(i)\right) p_{E}(i)+v(i), \quad i=1,2, \ldots, N
\end{aligned}
$$

A second stage identification is done since $C_{a_{p}}(\infty ; \beta)$ is only known approximately and the estimates of $a(\beta)$ and $b(\beta)$ are based on this a priori information. To update the damping term, a second variable is formed

$$
\begin{aligned}
z(t) & =C_{a}(t)-C_{a}(\infty ; \beta)-\eta(t) \\
& =\frac{\ell}{V} C_{a_{p}}(\infty ; \beta) p(t)
\end{aligned}
$$

If the measured values and new estimates for the unsteady term are substituted into Eq. (65), a new regression equation is obtained as

$$
z_{E}(i)=\frac{\ell}{V} C_{a_{p}}\left(\infty ; \beta_{E}(i)\right) p_{E}(i)+\varepsilon_{z}(i), \quad i=1,2, \ldots, N
$$

The model structure and parameters for the steady-flow damping term can be estimated using Eq. (66). This

\begin{tabular}{|c|c|}
\hline Parameter & Nonlinear Model \\
\hline$C_{l}(\infty ; \beta)$ & $\begin{array}{c}0.007+0.317 \beta+0.202 \beta^{2}-12.786 \beta^{3} \\
-2.371 \beta^{4}+91.123 \beta^{5}\end{array}$ \\
\hline$C_{l p}(\infty ; \beta)$ & $\begin{array}{c}-0.786-1.791 \beta^{2}+97.616 \beta^{4} \\
(0.0045) \quad(0.34) \quad(4.48)\end{array}$ \\
\hline$a(\beta)$ & $\begin{array}{ccc}0.527-43.668 \beta^{2}-507.228 \beta^{4} \\
(0.0051) & (0.39) & (5.096)\end{array}$ \\
\hline$b_{1}(\beta)$ & $\begin{array}{c}0.624-8.109 \beta^{2} \\
(0.011) \quad(0.14)\end{array}$ \\
\hline$\tau_{1}(\beta=0)$ & $\begin{array}{c}3.63 \\
(0.027) \\
\end{array}$ \\
\hline$R^{2}$ & 0.97 \\
\hline
\end{tabular}
completes the first iteration of the two stage identification. In the next iteration, $a(\beta)$ and $b(\beta)$ can be estimated again using the new values and model structure for the steady-flow damping term. This process is continued until parameter estimates have converged.

Table III. Nonlinear model for $C_{I}$ at $\alpha_{0}=37.5^{\circ}, \beta_{0}=0^{\circ}, \mathrm{F}-16 \mathrm{XL}, 2.5 \%$ scale.

Since harmonic analysis inferred that at least a cubic nonlinearity is present and the static component is a relatively large component in this case, a cubic representation of the static data was initially implemented. It was found, however, that a $5^{\text {th }}$ order polynomial was required for an adequate static model. Table III shows the final estimated model from the 2-stage process applied at $\alpha_{0}=37.5^{\circ}$ and the corresponding overall $R^{2}$. The parameter covariance matrix for this case contained some large pair-wise correlations indicating some identifiability issues. Figure 27 shows the measured and predicted rolling moments for the four frequencies considered. These graphs all show distortion from the regular elliptic shape associated with linear response. For each frequency, the nonlinear model prediction matches the response well. At the lowest frequency, $\mathrm{k}=0.066$, the distortion or nonlinearity is greatest, but the model match is still very good. Although the fit is very good the model in Table III is not completely satisfactory due to some inconsistency with small-amplitude linear analysis. Ideally the nonlinear model should represent the linear case as a subset then, for 
example, at $\beta=0$, the nonlinear model estimate, $C_{l p}(\infty ; \beta=0)$, should be close to the corresponding linear model analysis of the small amplitude data, a value of -0.13 . The differences may reflect a number of possible sources of error besides the model structure for the damping and unsteady terms, such as data accuracy and information content of the data.

The second example in this section considers data from two sources. First, measurements from a single-axis forcedoscillation experiment in roll with the NASA 5.5\% scale GTM, and second, simulated dynamic data representing the same experiment obtained using CFD. CFD was used to add insight into the investigation of roll instability found in the stall region. With the use of CFD best practices [62] researchers obtained corroborating data or investigate conditions not readily possible with ground or flight test facilities. The CFD solutions presented in this example were computed with the Reynolds Averaged Navier-Stokes equations using the Spalart-Allmaras turbulence model. The limitations of RANS for post-stall flows are well known, however, one objective was to assess the applicability of the RANS methodology in modeling the aggregate dynamic behavior of transport aircraft into and through stall. All solutions to follow, both static and dynamic, were advanced with second order physical time stepping.

Figure 28 provides a comparison of wind tunnel and CFD rolling moment coefficient results for a range of angle of attack and frequency. These results correspond to GTM roll forced sinusoidal oscillations with amplitude $20^{\circ}$. The oscillations were initiated from converged time-accurate static solutions at each angle of attack, and rotated for two complete oscillations. The dynamic solutions were essentially converged after one-fourth of a cycle. The $f=0.92 \mathrm{~Hz}$ solution was advanced with 360 time steps per roll cycle, and the $f=0.12$ and $0.06 \mathrm{~Hz}$ cases with 1800 time steps per cycle. The red lines on Fig. 28 indicate the second roll oscillation cycle of the CFD simulation and black corresponds to the wind tunnel mean-cycle measurements. Light gray lines show the wind tunnel measurements before averaging the cycles of oscillation. These measurements highlight the substantial measurement noise and other responses outside the desired bandwidth at high angles of attack where separated flows dominate. Overall, a reasonable match in response shape is presented although at $\alpha_{0}=12^{\circ}$ some differences occur reflecting the sensitivity of modeling this difficult unsteady flight condition.

To further explain the modeling sensitivity near the unstable and unsteady condition at $\alpha_{0}=12^{\circ}$, Fig. 29 presents streamlines over the wing that were computed to show how the flow is changing around this flight condition. These static solutions were advanced in time at a characteristic time step of 0.02, i.e., a free stream particle will travel the length of the mean aerodynamic chord in 50 time steps. In addition to streamlines, lift and pitching moment static curves are presented below the wings along with the corresponding wind tunnel data. Figure 29 shows a sequence of streamlines over the wing for four flight conditions: Fig. 29a, $\alpha=4^{\circ}$, showing normal flight conditions; Fig. 29b, $\alpha=$ $11.75^{\circ}$, showing conditions just before a break in the lift curve; Fig. 29c, $\alpha=12^{\circ}$, showing stalled conditions; and Fig. 29d, $\alpha=12.5^{\circ}$, showing post stall-break conditions. The figure shows that when the abrupt change in lift occurs at $\alpha$ $=12^{\circ}$, streamlines also make a rapid change and indicate a stalled wing tip. Also in this region, the lift curve slope has become flat on either side of the break in lift. These adverse conditions support the unstable roll damping found in harmonic analysis (Fig. 3) and is confirmed by roll divergence demonstrated in GTM subscale flight tests near this angle of attack. CFD results point to a more abrupt change in lift and pitching moment than found in the wind tunnel data.

CFD simulation was also used to generate detailed forced-oscillation data over a limited range of $\alpha_{0}$ in key regions of interest. Two regions in $\alpha_{0}$ were computed that covered $10^{\circ} \leq \alpha_{0} \leq 15^{\circ}$ and $24^{\circ} \leq \alpha_{0} \leq 30^{\circ}$ with fine $1^{\circ}$ increments. Figure 30 presents harmonic analysis for CFD simulated roll forced-oscillation data. To reduce clutter, only high frequency results from the wind tunnel analysis are overlaid for comparison. In general, the CFD analysis produced larger magnitudes for the coefficients but identified similar unsteady regions. The plots show that CFD results imply a small shift of the largest roll instability toward $\alpha_{0}=14^{\circ}$ rather than at $\alpha_{0}=12^{\circ}$ as indicated by the wind tunnel results. CFD results indicate unsteady behavior over a wider range of $11^{\circ} \leq \alpha_{0} \leq 15^{\circ}$, compared to the wind tunnel result that implied the behavior exists only near at $\alpha_{0}=12^{\circ}$. In the lower $\alpha_{0}$ range $R^{2}$ analysis is similar to the wind tunnel results. In that range both wind tunnel and CFD capture the nonlinearity at high frequency for $\alpha_{0}=14^{\circ}$. At the upper $\alpha_{0}$ range, the CFD results present higher values of $R^{2}$ reflecting the lower contribution of noise and other higher order effects in CFD simulation and confirming the use of linear unsteady models.

Parameter estimation using $\mathrm{OE}$ in the time domain and linear model equations Eq. (21) was applied to forced oscillation wind tunnel data [63]. Measured inputs, $\dot{\beta}$, and outputs, $C_{l}$, for each of the frequencies tested were included in the time domain estimation process. Results from application of OE to the roll forced-oscillation data is shown in Fig. 31. This figure shows the estimated parameters and their $2 \sigma$ bounds for a large range of angle of attack, for $0^{\circ} \leq \alpha_{0} \leq 70^{\circ}$. Uncertainty bounds are generally very small over the range of angle of attack considered. This implies good information content or that adequate sensitivity was obtained for parameter estimation using the wind 
tunnel data. The top plot shows steady-flow damping. Consistent with previous results, this term is neutral to unstable at $\alpha_{0}=12^{\circ}$ and $\alpha_{0} \geq 50^{\circ}$. The middle plot shows model parameter, $a$, that acts as a gain on the unsteady term and is, in general, relatively small except at $\alpha_{0}=12^{\circ}$. The bottom plot presents a non-dimensional time constant defining lag in the aerodynamic response. This plot shows three regions where unsteady behavior is present and the relative size of the lags in those regions. The smallest lag or fastest response is at $\alpha_{0}=12^{\circ}$.

As an example of model fidelity, at $\alpha_{0}=30^{\circ}$, response prediction is presented in Fig. 32. This example shows measured and unsteady model responses for both low and high frequency cases of the rolling moment coefficient against sideslip. Response measurements plotted against $\phi$ are commonly used and have been presented in this study. However for this graphic, using $\beta$ highlights the adequacy of the linear unsteady model even with relatively large displacements of this key state variable. The estimated linear static curve is included to provide a reference line. Since linear models are used, the computed responses are regular ellipses. In general the models represent the measured data very well. One notable feature for the low frequency case is the very low signal-to-noise for this test point. At the higher frequency, signal-to-noise is improved but still not very good. The measured data for the high frequency case does reflect some nonlinearity as indicated by the slightly irregular elliptical shape of the data; however, the linear model is still an adequate representation in this case. Although lag in the response caused by the unsteady flow is not readily visible in this type of plot, some evidence is presented by the rotation of the ellipse relative to the static line. For a linear model without a lag in the response, the long axis of the ellipse will align with the static line.

\section{Concluding Remarks}

Aerodynamic research has a long history addressing the nonlinear unsteady modeling problem both analytically and experimentally. This paper reviewed a portion of that history and highlighted contributions made at the NASA Langley Research Center, primarily covering the last decade. Collaboration between analytical and experimental researchers offers a significant opportunity to further the technology in both disciplines. When results from these two areas are compared, model identification and validation methods should be carefully addressed to ensure successful and productive collaborations. This paper offers a methodology for comparing models obtained by applying system identification methods to CFD simulations and wind tunnel measurements. The methodology can accommodate general unsteady aerodynamic behaviors and characterize model uncertainty beyond conventional comparisons and aerodynamic modeling methods.

\section{Acknowledgments}

Support was provided by several NASA programs, however primary support was provided by the NASA Aviation Safety Program.

\section{References}

[1] Byran, G. H.: Stability in Aviation, MacMillan and Co., London, 1911.

[2] Klein, V., Batterson, J.G., Murphy, P.C.: Determination of Airplane Model Structure from Flight Data by Using Modified Stepwise Regression, NASA TP 1916, 1981.

[3] Tobak and Schiff: On the Formulation of the Aerodynamic Characteristics in Aircraft Dynamics, NASA TR R-456, 1976.

[4] Goman, M.G., Stolyaraov, G. I., Tyrtyshnikov, S.L., Usolcev, S.P., and Khrabrov, A.N.: Mathematical Description of Longitudinal Aerodynamic Characteristics at High Angles of Attack Accounting for Dynamic Effects of Separated Flow. TsAGI Preprint No. 9, 1990 (in Russian).

[5] Goman, M. and Khrabrov, A.(TsAGI): State-Space Representation of Aerodynamic Characteristics of an Aircraft at High Angles of Attack. Journal of Aircraft, Vol.31, No.5, 1994, pp. 1109-1115.

[6] Goman, M., Khrabrov, A., and Usolcev, S.: Unsteady Aerodynamic Model for Large Amplitude Maneuvers and its Parameter Identification. $11^{\text {th }}$ IFAC Symposium on Identification, July 8-11, 1997, Kitakyushu, Japan, Vol. 1, pp.399-403.

[7] Fishenberg, D.: Identification of an Unsteady Aerodynamic Stall Model from Flight Test Data. AIAA Paper 95-3438-P, 1996.

[8] Klein, V., and Noderer, K.D.: Modeling of Aircraft Unsteady Aerodynamic Characteristics”, Part I - Postulated Models, NASA TM 109120, May, 1994; Part 2 - Parameters Estimated From Wind Tunnel Data, NASA TM 110161, April 1995; Part 3 - Parameters Estimated From Flight Data, NASA TM 110259, May 1996.

[9] Klein, V., Murphy, P.C., Curry, T.J., and Brandon, J.M.: Analysis of Wind Tunnel Longitudianl Static and Oscillatory Data of the F-16XL Aircraft, NASA/TM-97-206276, December 1997.

[10] Gupta, N. K., and Iliff, K. W.: Identification of Aerodynamic Indicial Functions Using Flight Test Data, AIAA Paper 19821375, August 1982.

[11] Jones, Robert T. and Fehlner, Leo F., “Transient Effects of the Wing Wake on the Horizontal Tail,” NACA TN-771, 1940.

[12] Tobak, Murray, "On the Use of Indicial Function Concept in the Analysis of Unsteady Motions of Wings and Wing-Tail Combinations, NACA Rep. 1188, 1954. 
[13] Klein, Vladislav, "Modeling of Longitudinal Unsteady Aerodynamics of a Wing-Tail Combination,” NASA CR-1999209547, September, 1999.

[14] Myatt, James, H.: Modeling the Rolling 65-Degree Delta Wing with Critical State Encounters, AIAA 97-3646, August 1997.

[15] Chin, S., and Lan, E.: Fourier Functional Analysis for Unsteady Aerodynamic Modeling. AIAA Paper 91-2867-CP, 1991.

[16] Reisenthel, P. H.: Application of Nonlinear Indicial Modeling to the Prediction of a Dynamically Stalling Wing. AIAA 962493, August, 1996.

[17] Klein, V., Murphy, P.C.: Estimation of Aircraft Nonlinear Unsteady Parameters From Wind Tunnel Data, NASA TM-1998208969, December 1998.

[18] Murphy, P.C., Klein, V.: Estimation of Aircraft Unsteady Aerodynamic Parameters From Dynamic Wind Tunnel Testing, AIAA Paper 2001-4016, August 2001.

[19] Murphy, P.C., Klein, V.: Validation of Methodology for Estimating Aircraft Unsteady Aerodynamic Parameters from Dynamic Wind Tunnel Tests, AIAA 2003-5397, August, 2003, (AFM best paper).

[20] Brunton, Steven L., and Rowley, Clarence W., "Low-dimensional State-space Representations for Classical Unsteady Aerodynamic Models,” AIAA 2011-476, January 2011.

[21] Murphy, Patrick C., and Landman, Drew, "Experiment Design for Complex VTOL Aircraft with Distributed Propulsion and Tilt Wing,” AIAA 2015-0017, January 2015.

[22] Busan, Ronald C., Rothhaar, Paul M., Croom, Mark A., Murphy, Patrick C. Grafton, Sue B., and O-Neal, Anthony W., “Enabling Advance Wind-Tunnel Research Methods Using NASA Langley 12-Foot Low Speed Tunnel,” AIAA 2014-3000, June 2014.

[23] Rothhaar, Paul M., Murphy, Patrick C., Bacon, Barton J., Gregory, Irene M., Grauer, Jared, A., Busan, Ronald C., and Croom, Mark A., "NASA Langley Distributed Propulsion VTOL Tilt-Wing Aircraft Testing, Modeling, Simulation, Control, and Flight Test Development,” AIAA 2014-2999, June 2014

[24] Klein, Vladislav, Murphy, P.C., and Szyba, Nathan M., “Analysis of Wind Tunnel Lateral Oscillatory Data of the F-16XL Aircraft,” NASA/TM-2004-213246, 2004.

[25] Abramov, N. B., Goman, M. G., Greenwell, D. J., and Khrabrov, A. N.: Two-Step Linear Regression for Identification of High Incidence Unsteady Aerodynamic Model. AIAA Paper 2001-4080, 2001.

[26] Murphy, P.C. and Klein, V., “Transport Aircraft System Identification from Wind Tunnel Data”, AIAA 2008-6202, August 2008.

[27] Murphy, P.C. and Klein, V., “Transport Aircraft System Identification Using Roll and Yaw Oscillatory Wind Tunnel Data”, AIAA 2010-8122, August 2010.

[28] Murphy, Patrick C. and Klein, Vladislav, "Estimation of Longitudinal Unsteady Aerodynamics of a Wing-Tail Combination from Wind Tunnel Data,” AIAA Atmospheric Flight Mechanics Conference, AIAA 2006-6154, AIAA Washington, DC, 2006.

[29] Murphy, Patrick C. and Klein, Vladislav, "On Problems Associated with Modeling Wing-Tail Configurations from Wind Tunnel Data,” AIAA Atmospheric Flight Mechanics Conference, AIAA 2007-6722, AIAA Washington, DC, 2007.

[30] Morelli, Eugene A., and Grauer, Jared A., "Efficient Flight Testing and Modeling Techniques for Full-Envelope Flight in Adverse Conditions,” to be published JGCD, 2016.

[31] Murphy, Patrick C., Vicroy, Dan D., Kramer, Brian, and Kerho, Michael, "Hybrid Wing Body Model Identification Using Forced-Oscillation Water Tunnel Data,” AIAA Atmospheric Flight Mechanics Conference, AIAA 2014-2197, June, 2014.

[32] Murphy, Patrick C., Klein, Vladislav, and Szyba, Nathan M., "Progressive Aerodynamic Model Identification From Dynamic Water Tunnel Test of the F-16XL Aircraft,” AIAA Atmospheric Flight Mechanics Conference, AIAA 2004-5277, AIAA Washington, DC, 2004.

[33] Murphy, Patrick C. and Klein, Vladislav, "Estimation of Unsteady Aerodynamic Models from Dynamic Wind Tunnel Data,” RTO AVT-189 Specialists Meeting, Portsdown West, UK, October 12-14, 2011. NATO RTO 2011 Paper No. MP-AVT-18920.

[34] Bommanahal, Mallesh. and Goman Mikhail: Nonlinear Unsteady Aerodynamic Modeling by Volterra Variational Approach, AIAA 2012-4654, August, 2012.

[35] Klein, Vladislav and Morelli, Eugene, “Aircraft System Identification: Theory and Practice,” 1st edition, AIAA Inc., Reston, Virginia, 2006.

[36] Jordan, T. L., and Bailey, "NASA Langley’s AirSTAR Testbed: A Subscale Flight Test Capability for Flight Dynamics and Control Systems," AIAA-2008-6660, August 2008.

[37] Morelli, Eugene A. and De Loach, Richard, "Wind Tunnel Database Development Using Modern Experiment Design and Multivariate Orthogonal Functions,” AIAA 2003-0653, January 2003.

[38] Morrison, Joseph H., and Hemsch, Michael J., "Statistical Analysis of CFD Solutions from the Third AIAA Drag Prediction Workshop,” AIAA 2007-254, January 2007.

[39] Hemsch, Michael J., "Statistical Analysis of Computational Fluid Dynamics Solutions from the Drag Prediction Workshop," Journal of Aircraft, Vol. 41, No. 1, January-February, 2004.

[40] Vicroy, D. and Loeser, T., “SACCON Dynamic Wind Tunnel Tests at DNW- NWB and 14'x22' NASA LaRC”, AIAA-20104394, June 2010.

[41] Murphy, Patrick C, Klein, Vladislav, Frink, Neal T., and Vicroy, Daniel D, "System Identification Applied to Dynamic CFD Simulation and Wind Tunnel Data”, AIAA 2011-6522, August 2011. 
[42] Landman, Drew, Simpson, Jim, Vicroy, Dan D., and Parker, Peter, "Response Surface Methods for Efficient Complex Aircraft Configuration Aerodynamic Characterization,” Journal of Aircraft, Vol. 44, No. 4, July-August 2007.

[43] Landman, Drew, Simpson, Jim, Vicroy, Dan D., and Parket, Peter, "Hybrid Design for Aircraft Wind-Tunnel Testing Using Response Surface Methodologies,” Journal of Aircraft, Vol. 44, No. 4, July-August 2007.

[44] DeLoach, Richard, and Erickson, G. E., "Low-Order Response Surface Modeling of Wind Tunnel Data Over Truncated Inference Subspaces,” AIAA 2003-0456, January 2003.

[45] DeLoach, Richard, "Response Surface Modeling Tolerance and Inference Error Risk Specifications: Proposed Industry Standards,” AIAA 2012-2859, June 2012.

[46] DeLoach, Richard, "Propagation of Computational Uncertainty Using the Modern Design of Experiments,” NASA Technical Report, http://ntrs.nasa.gov/archive/nasa/casi.ntrs.nasa.gov/20080002264.pdf, 2008.

[47] Montgomery, Douglas C., “Design and Analysis of Experiments,” $5^{\text {th }}$ edition, John Wiley \& Sons, 2001.

[48] Pattinson, J., Lowenberg, M. H., and Goman, M. G., "Multi-Degree-of-Freedom Wind-Tunnel Maneuver Rig for Dynamic Simulation and Aerodynamic Model Identification”, Journal of Aircraft, Vol. 50, No. 2, March-April 2013, DOI: 10.2514/1.C031924.

[49] Cummings, R. and A. Schütte, A., "Integrated Computational/Experimental Approach to Unmanned Combat Air Vehicle Stability \& Control Estimation”, Journal of Aircraft, Vol. 49, No. 6 (2012), pp.1542-1557. doi: 10.2514/1.C031430.

[50] Loeser, T., Vicroy, D., and Schütte, A., “SACCON Static Wind Tunnel Tests at DNW- NWB and 14'x22' NASA LaRC”, AIAA-2010-4393, June 2010.

[51] Gilliot, A., “Static and Dynamic SACCON PIV Tests - Part I: Forward Flowfield”, AIAA-2010-4395, June 2010.

[52] Konrath, R., Roosenboom, E., Schröder, A., Pallek, D., and Otter, D., “Static and Dynamic SACCON PIV Tests - Part II: Aft Flow Field”, AIAA-2010-4396, June 2010.

[53] Frink, N.T., "Strategy for Dynamic CFD Simulations on SACCON Configuration”, AIAA 2010-4559, June 2010.

[54] Vallespin, D., Boelens, O. and Badcock, K., "SACCON CFD Simulations Using Structured Grid Approaches”, AIAA-20104560, June 2010.

[55] Tormalm, M. Tomac, M. and Schmidt, S., “Computational Study of Static And Dynamic Vortical Flow over the Delta Wing SACCON Configuration Using the FOI Flow Solver Edge”, AIAA-2010-4561, June 2010.

[56] Le Roy, J. “SACCON CFD Static and Dynamic Derivatives Using elsA”, AIAA-2010-4562, June 2010.

[57] Chakravarthy S. and Chi, D., "SACCON CFD Simulations Using CFD++", AIAA-2010-4563, June 2010.

[58] Schütte, A., Hummel, D. and Hitzel, S. "Numerical and Experimental Analyses of the Vortical Flow Around the SACCON Configuration”, AIAA-2010-4690, June 2010.

[59] Cummings, R. Petterson, K. Jirasek, A. and Schmidt, S., "SACCON Static and Dynamic Motion Flow Physics Simulations Using COBALT”, AIAA-2010-4691, June 2010.

[60] Cummings, R.M., and Schütte, A., “The NATO STO Task Group AVT-201 on 'Extended Assessment of Stability and Control Prediction Methods for NATO Air Vehicles,” AIAA 2014-2000, June 2014.

[61] Rohlf, D., Schmidt, S., and Irving, J., "SACCON Stability and Control Analysis Applying System Identification Techniques”, AIAA 2010-4399, June 2010.

[62] Thompson, J. R., Frink, Neal T., Murphy, Patrick C., "Guidelines for Computing Longitudinal Dynamic Stability Characteristics of a Subsonic Transport,” AIAA 2010-4819, June 2010.

[63] Murphy, Patrick C, Klein, Vladislav, and Frink, Neal T., "Unsteady Aerodynamic Modeling in Roll for the NASA Generic Transport Model”, AIAA 2012-4652, August 2012.

[64] Frink, Neal T., Murphy, Patrick C, Atkins, Harold L., Viken, Sally A.,Petrilli, Justin L., Gopalarathnam, Ashok, and Paul, Ryan C., "Status of Computational Aerodynamic Modeling Tools for Aircraft Loss-of-Control," to be published JGCD, 2016.

[65] Williams, Brianne Y., Landman, Drew, Flory IV, Isaac L., and Murphy, Patrick C., "The Effect of Systematic Error in Forced Oscillation Testing”, AIAA 2012-0768, January 2012.

[66] Pototzky, Anthony S., and Murphy, Patrick C., "Roll Damping Derivatives from Generalized Lifting-Surface Theory and Wind Tunnel Forced-Oscillation Tests”, AIAA 2014-0731, January 2014.

[67] Klein, Vladislav, Murphy, Patrick C., Curry, Timothy J., Brandon, Jay M.: Analysis of Wind Tunnel Longitudinal Static and Oscillatory Data of the F-16XL Aircraft, NASA TM-1997-206276, December, 1997.

[68] Ghoreyshi, M., Frink, N.T., van Rooij, M., Lofthouse, A.J., Cummings, R.M., Nayani, S., “Collaborative Evaluation of CFDto-ROM Dynamic Modeling”, to be presented at AIAA Science and Technology Forum and Exposition (SciTech 2016), San Diego, California, January 4-8, 2016. 
Figures
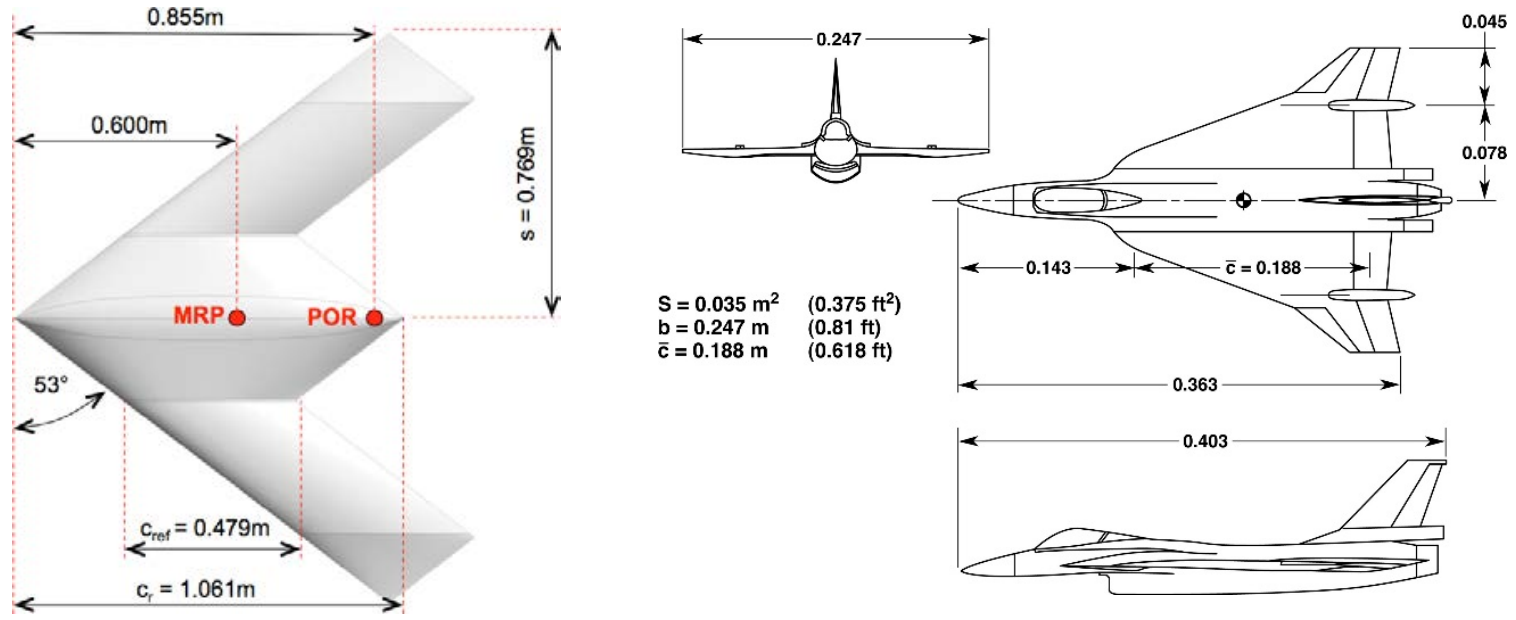

$\mathrm{S}=0.77 \mathrm{~m}^{2}, \bar{c}=\mathbf{0 . 4 7 9} \mathrm{m}, \mathrm{b} / \mathbf{2}=\mathbf{0 . 7 6 9}$

Figure 1a. Model geometry for SACCON sub-scale aircraft, Ref. [39].

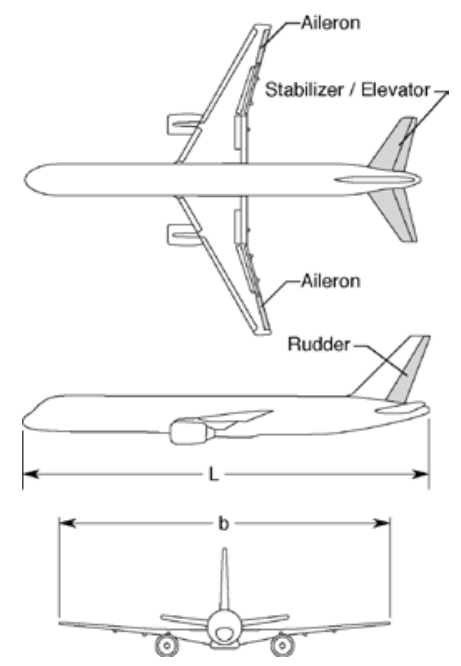

$\mathrm{S}=5.90 \mathrm{ft}^{2}, \bar{c}=0.915 \mathrm{ft}, \mathrm{b}=6.85 \mathrm{ft}$

Figure 1c. Model geometry for NASA experimental sub-scale aircraft.
Figure 1b. Three-view drawing of 2.5\% F-16XL water tunnel model. 


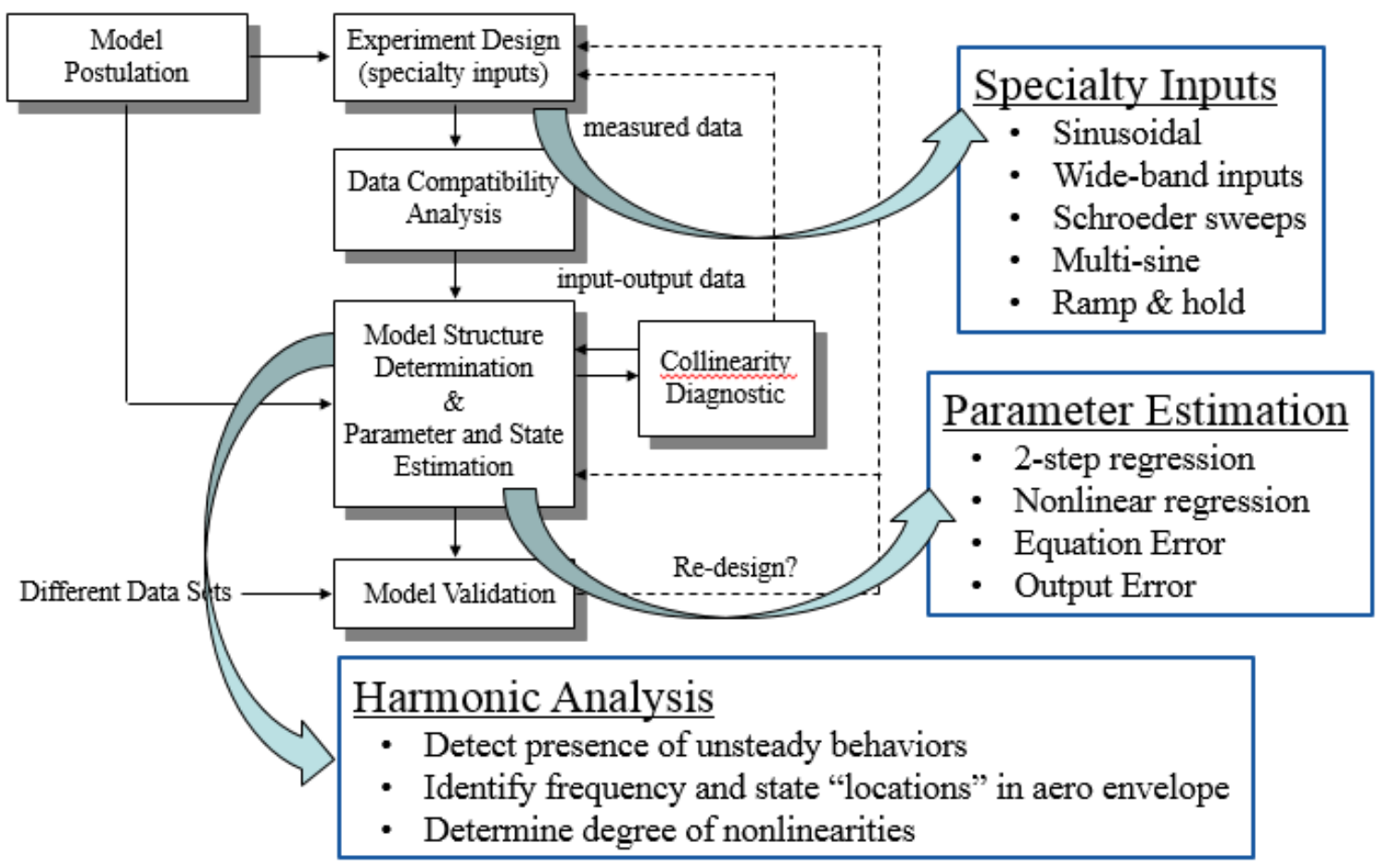

Figure 2. System Identification model for unsteady modeling applications.
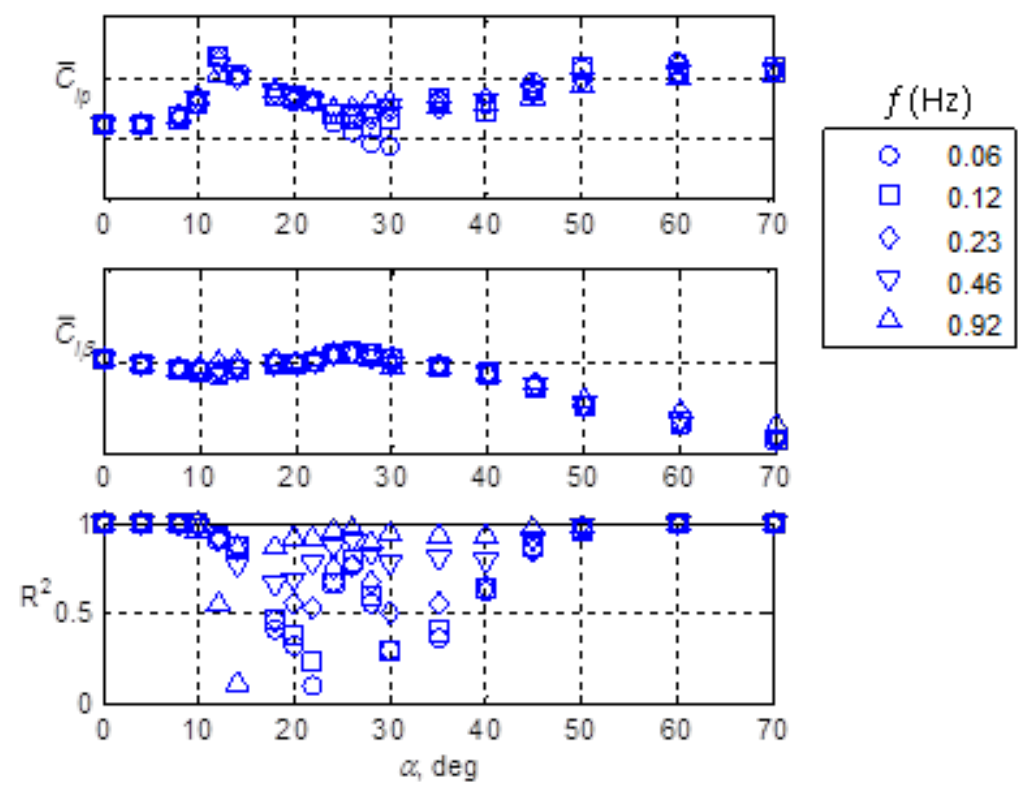

Figure 3. Harmonic analysis for rolling moment coefficient, roll oscillations, $\phi_{A}=20^{\circ}$, NASA GTM configuration. 

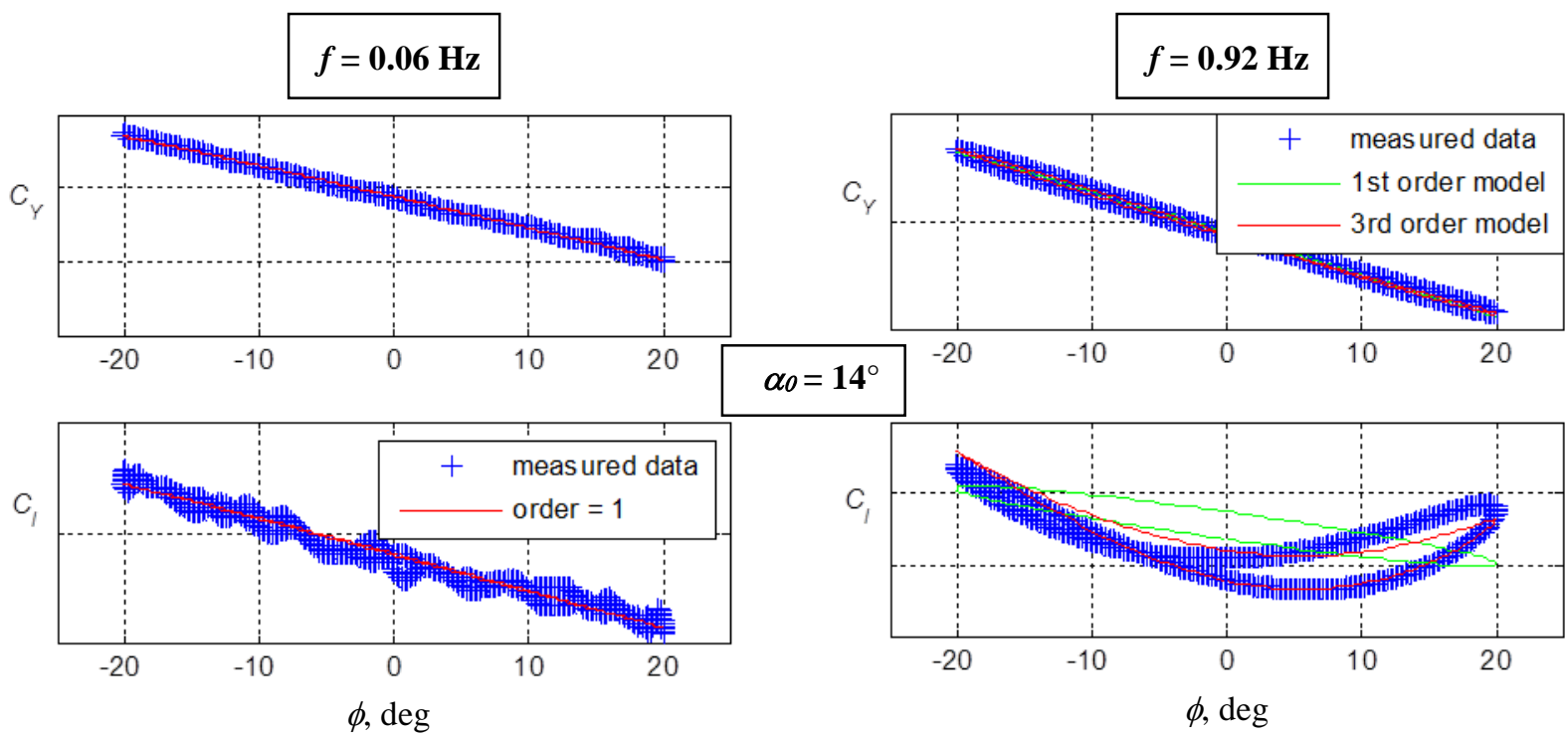

Fig. 4a Mean cycle, first-order, and $3^{\text {rd }}$-order harmonic model responses, $\alpha_{0}=14^{\circ}$, amplitude $\phi_{A}=20^{\circ}$, NASA GTM configuration.

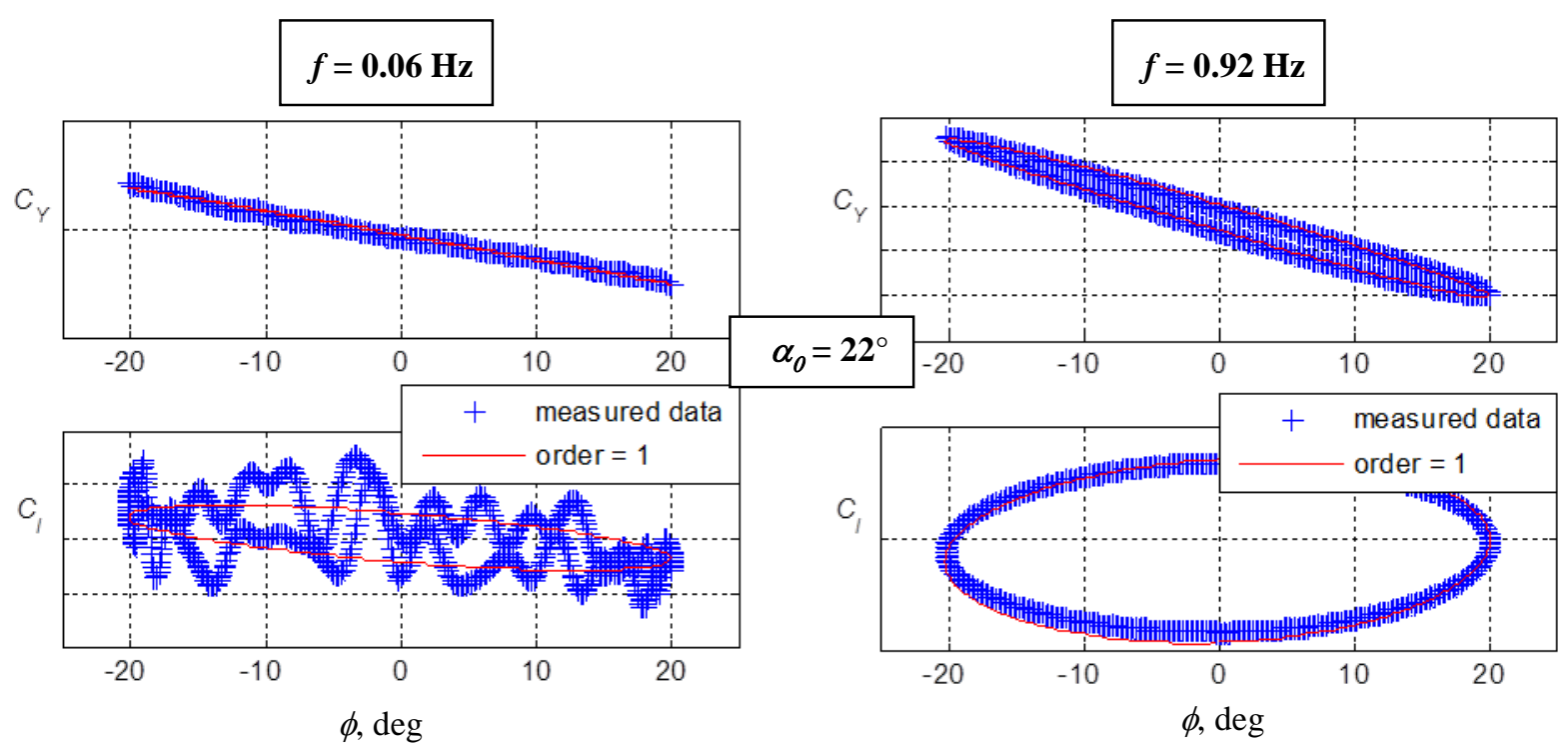

Fig. $4 \mathrm{~b}$ Mean cycle and first-order harmonic model responses, $\alpha_{0}=22^{\circ}$, amplitude $\phi_{A}=20^{\circ}$, NASA GTM configuration. 


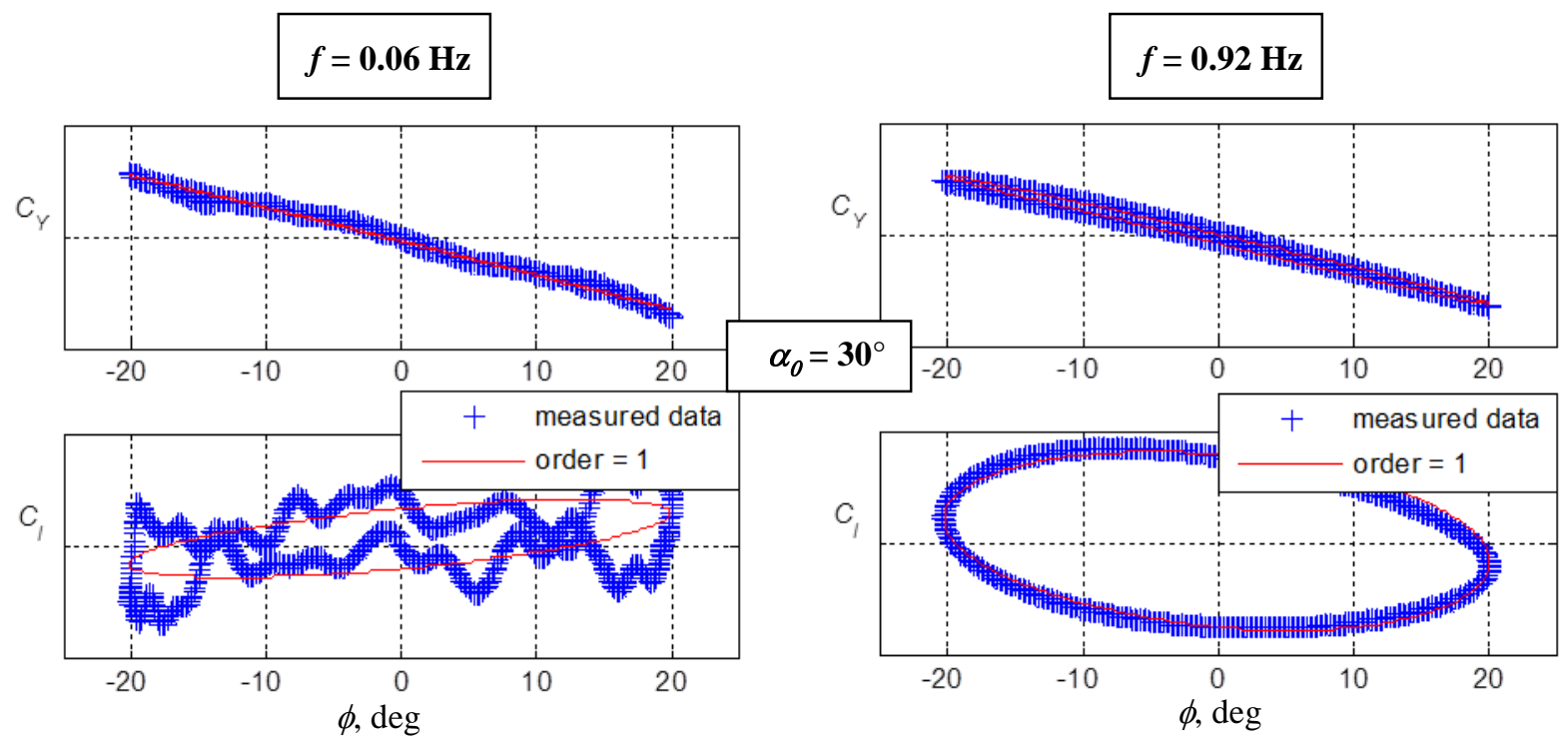

Fig. 4c Mean cycle and first-order harmonic model responses, $\alpha_{0}=30^{\circ}$, amplitude $\phi_{A}=20^{\circ}$, NASA GTM configuration.

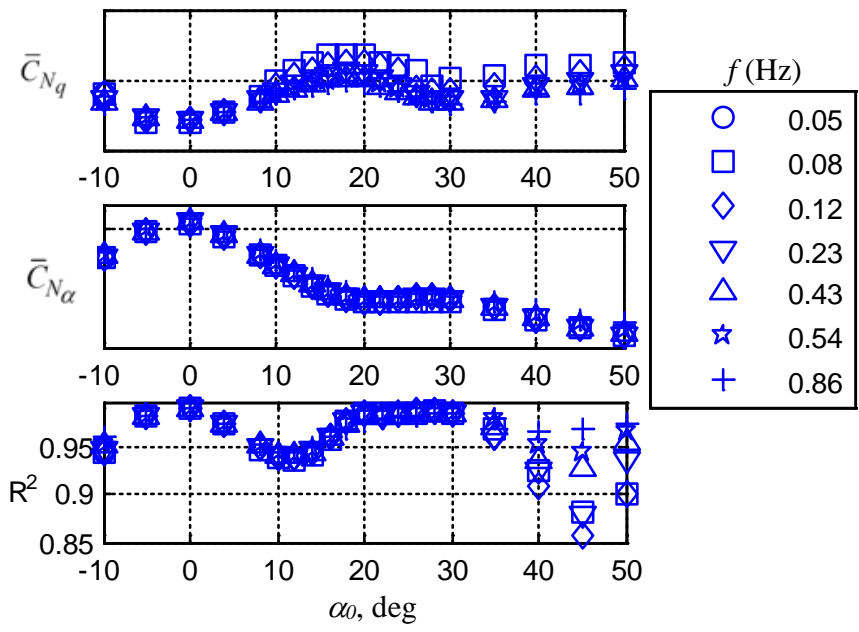

Figure 5. Harmonic analysis for normal-force coefficient, NASA GTM configuration, $\alpha_{A}=10^{\circ}$.

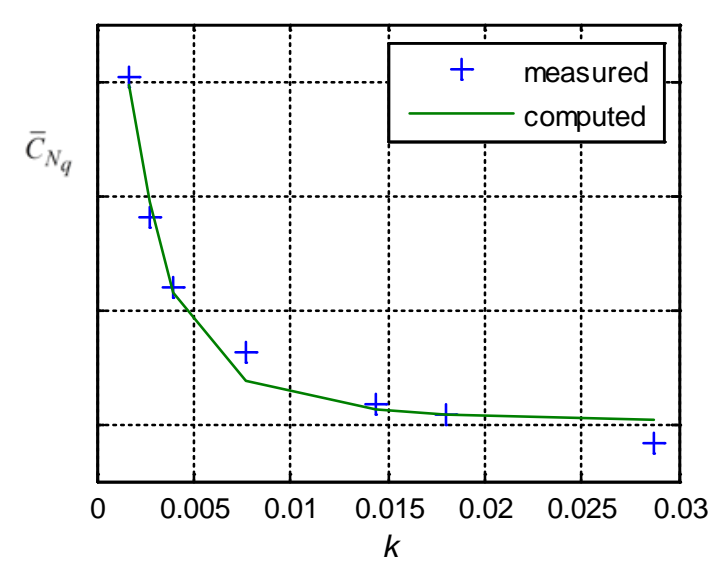

Figure 6. Results of nonlinear regression for measured out-of-phase components of NASA GTM, $\alpha_{0}=18^{\circ}, \alpha_{A}=10^{\circ}$. 


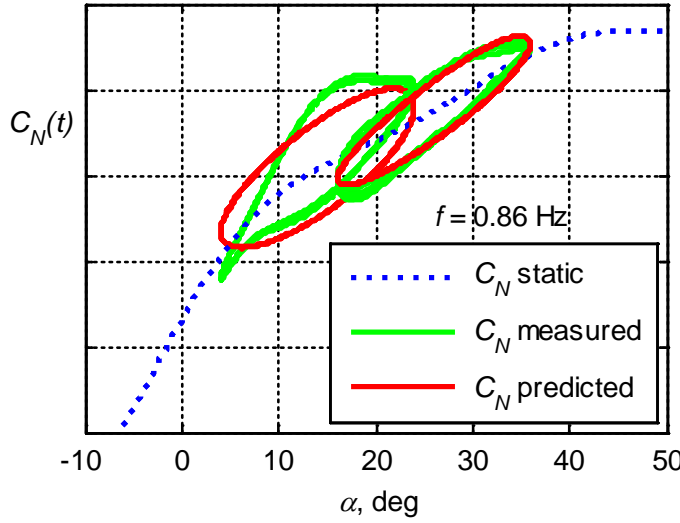

Figure 7. Comparison of measured and predicted normal-force coefficient, NASA GTM, $f=0.86 \mathrm{~Hz}$, $\alpha_{A}=10^{\circ}$, with oscillations about $\alpha_{0}=\left(14^{\circ}, 26^{\circ}\right)$.

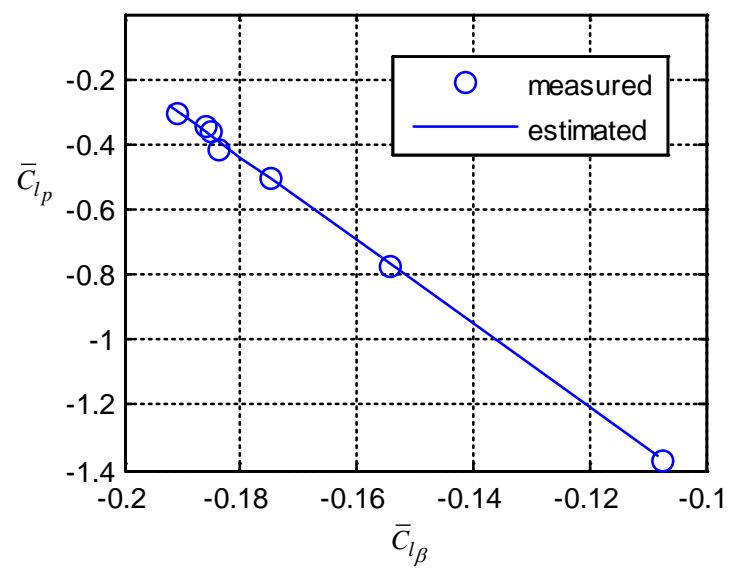

Figure 9. Measured vs Estimated rollmoment coefficients, $18 \%$ F-16XL, $\phi_{A}=10^{\circ}$.

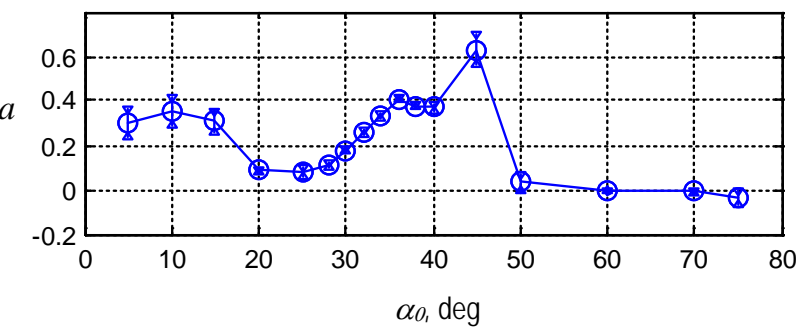

Figure 10b. Two-Step Regression estimates, 18\% F-16XL, $\phi_{A}=10^{\circ}$.

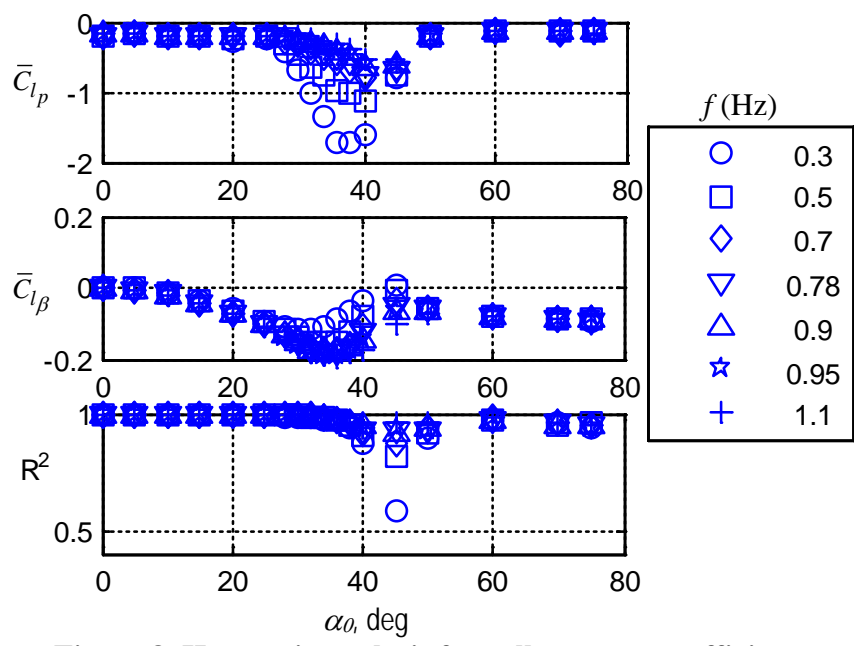

Figure 8. Harmonic analysis for roll-moment coefficient, $18 \%$ F-16XL configuration, $\phi_{A}=10^{\circ}$.
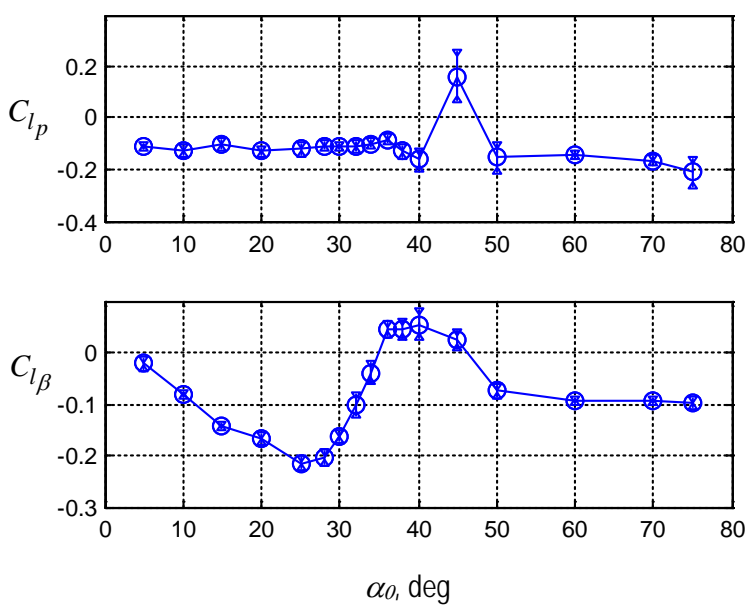

Figure 10a. Two-Step Regression estimates, $18 \%$ F16XL, $\phi_{A}=10^{\circ}$.

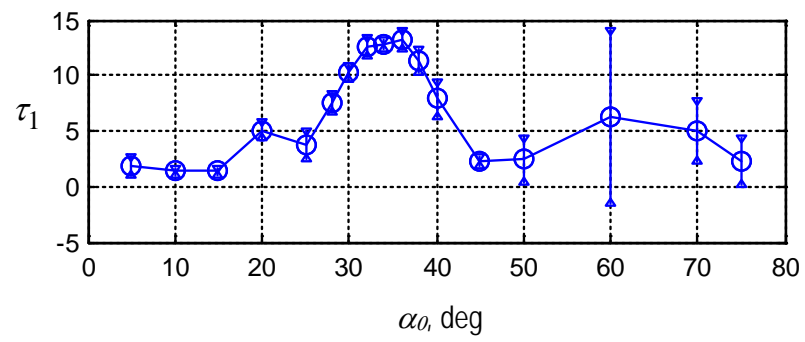

Figure 10c. Two-Step Regressıon estimates, $18 \%$ F16XL, $\phi_{A}=10^{\circ}$. 

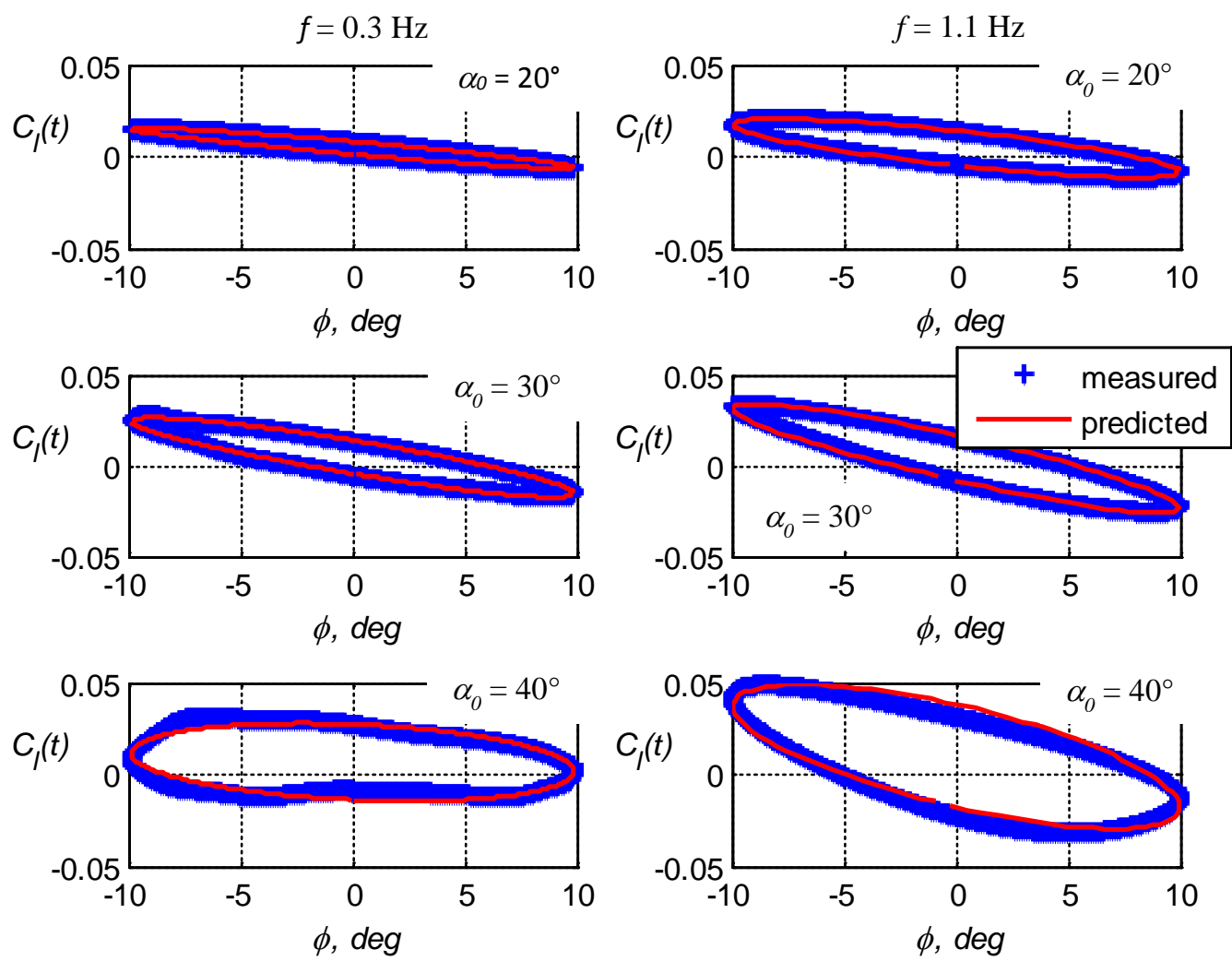

Figure 11. Two-Step Regression model of roll-moment predicted and measured data, $18 \% \mathrm{~F}-16 \mathrm{XL}, \phi_{A}=10^{\circ}$.

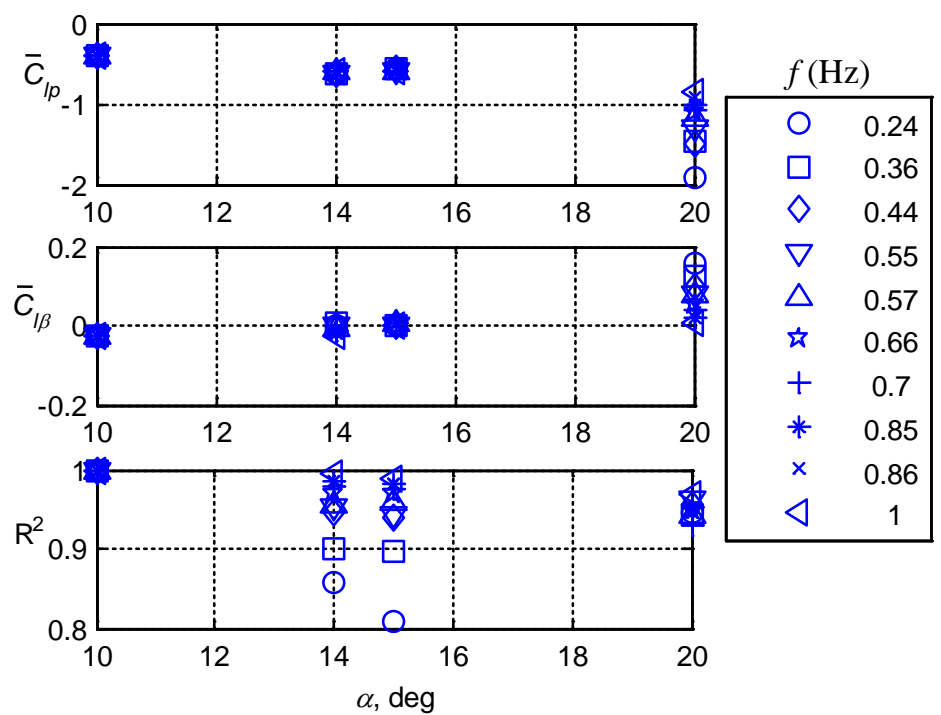

Figure 12. Roll moment out-of-phase and in-phase components from harmonic analysis of wind tunnel forced oscillations in roll, $\phi_{A}=5^{\circ}$. SACCON. 


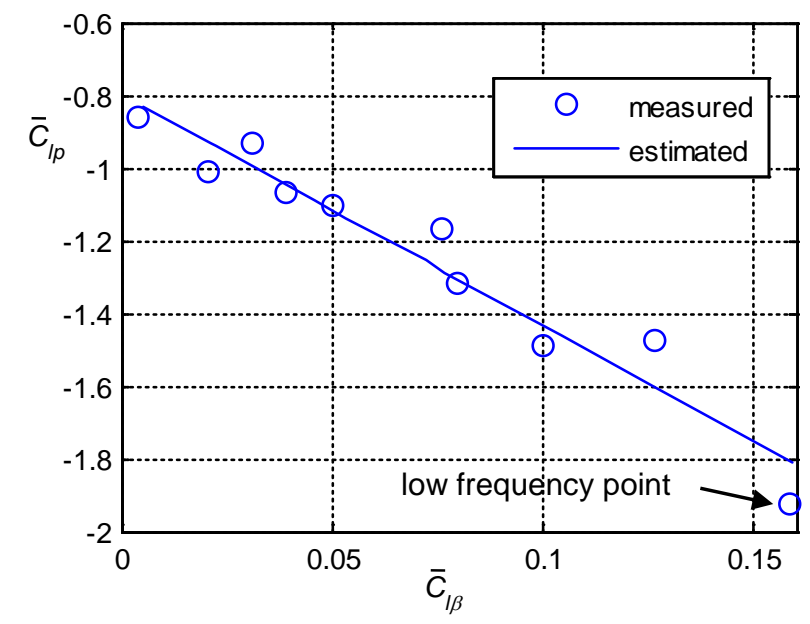

Figure 13. Two-step LR model estimates from roll oscillatory wind tunnel data at $20^{\circ}$ angle of attack, $\phi_{A}=5^{\circ}$. SACCON.

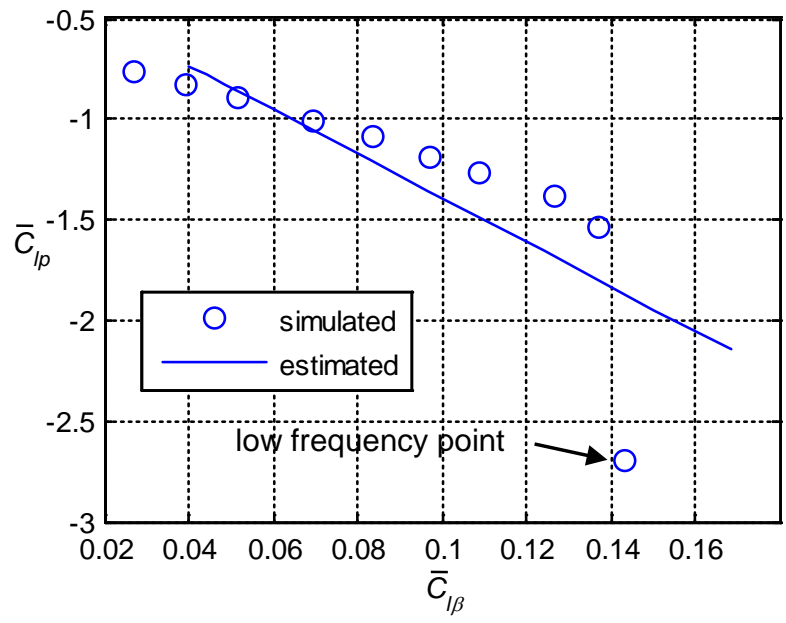

Figure 14. Out-of-phase vs in-phase component at $20^{\circ}$ angle of attack. CFD roll oscillatory data using SA turbulence model, $\phi_{A}=5^{\circ}$. SACCON.

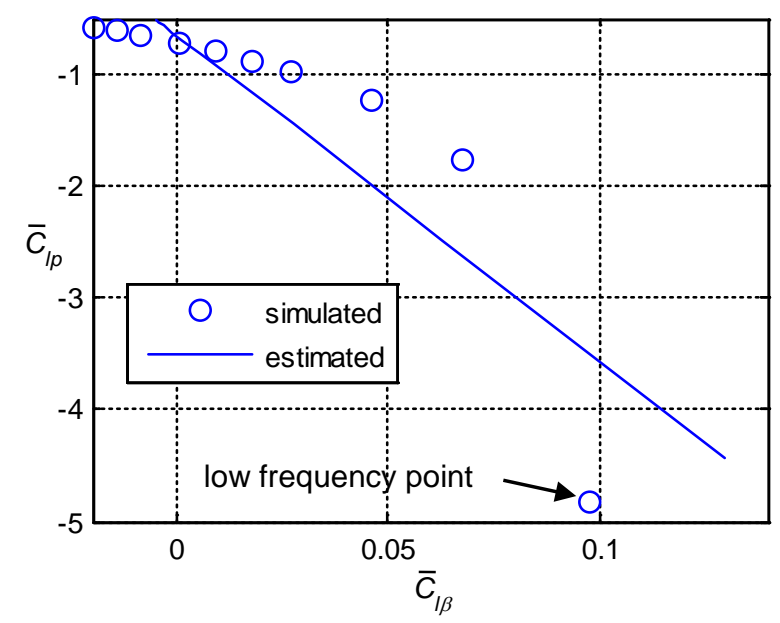

Figure 15. Out-of-phase vs in-phase component at $20^{\circ}$ angle of attack. CFD roll oscillatory data using SST turbulence model, $\phi_{A}=5^{\circ}$. SACCON. 

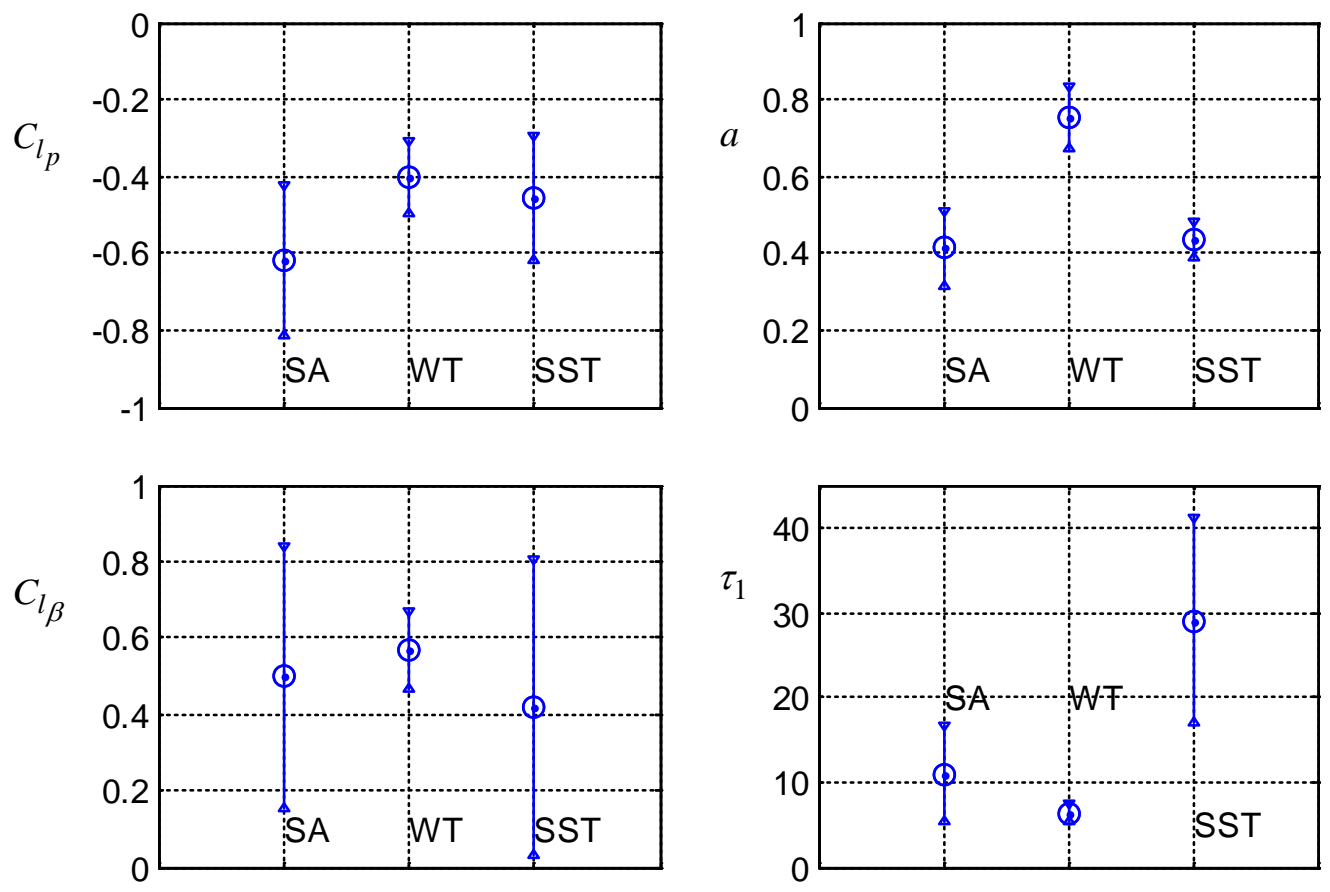

Figure 16. Two-step LR model parameter estimates and $2 \sigma$ error bounds, at $\alpha_{0}=20$, using wind tunnel (WT) and CFD roll oscillatory data, $\phi_{A}=5^{\circ}, \mathrm{SACCON}$.
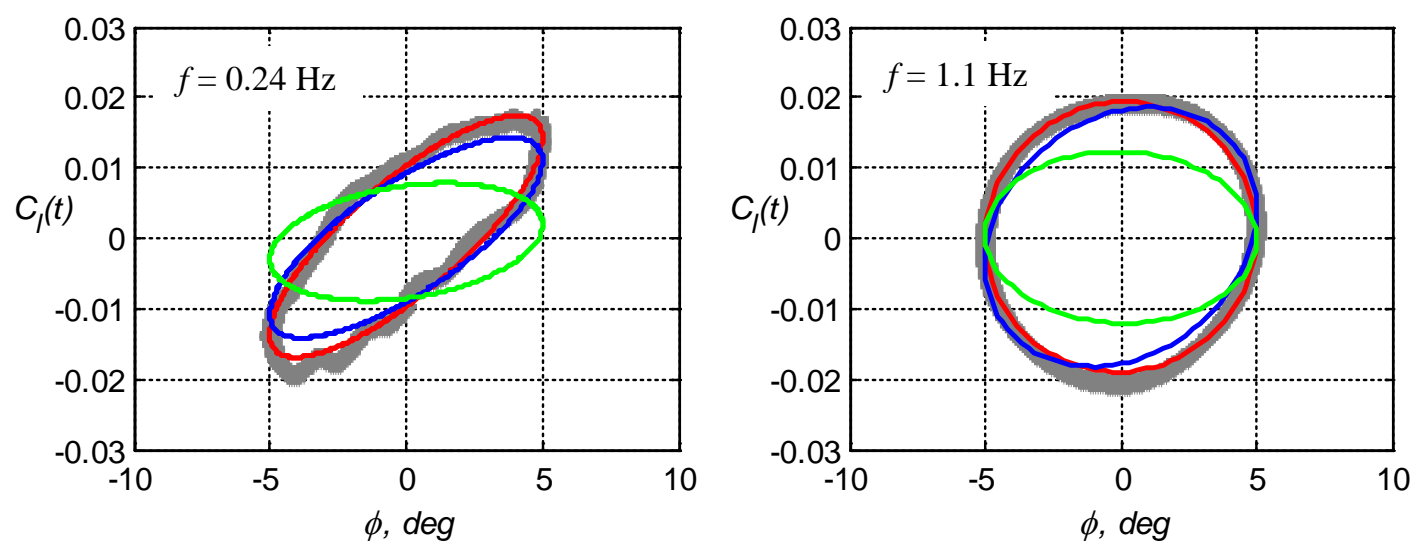

Figure 17. Model response predictions at $\alpha o=20^{\circ}$ for roll oscillatory data, $\phi_{A}=5^{\circ}, \mathrm{SACCON}^{3}$. Key: Gray = wind tunnel measurements, Red = Two-Step model from tunnel measurements, Blue = Two-Step model from CFD-SA, Green = Two-Step model from CFD-SST . 

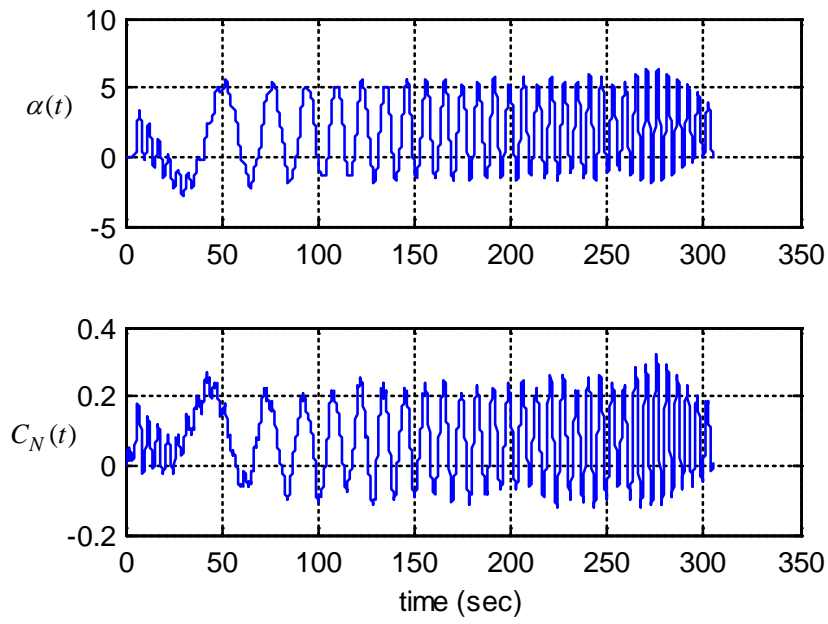

Figure 18. Measurements of $\alpha$ perturbations from $\alpha_{0}=42.5^{\circ}$ and normal force during wide-band experiment with $2.5 \%$ F-16XL in water tunnel.

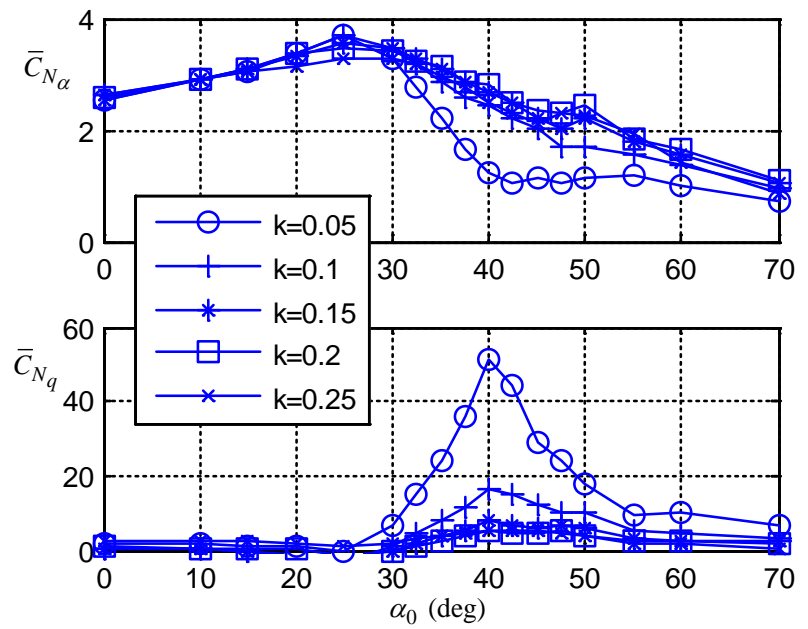

Figure 20. In-phase and out-of-phase coefficients from wide-band experiments, $2.5 \% \mathrm{~F}-16 \mathrm{XL}, \alpha_{A}=5^{\circ}$.

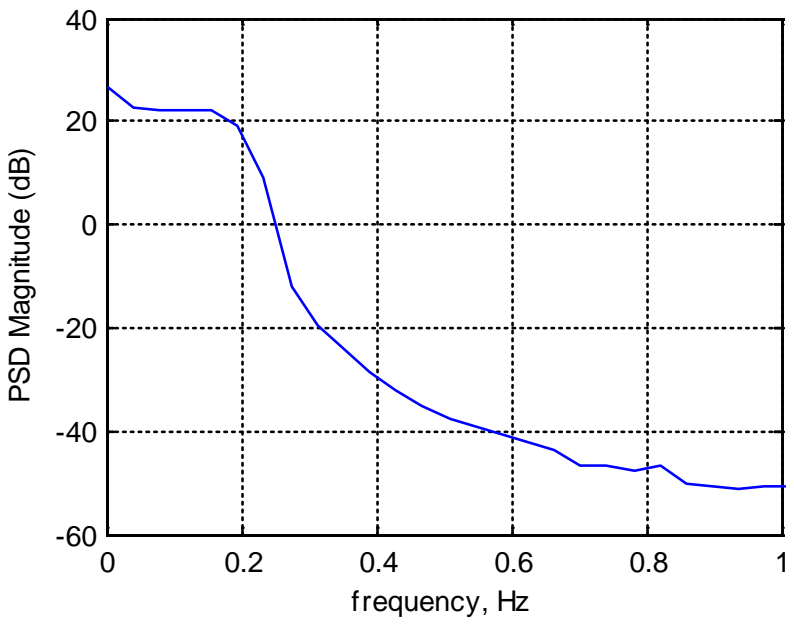

Figure 19. Harmonic content of $\alpha$ wide-band input, $2.5 \% \mathrm{~F}-16 \mathrm{XL}$ in water tunnel, $\alpha_{A}=5^{\circ}$. 

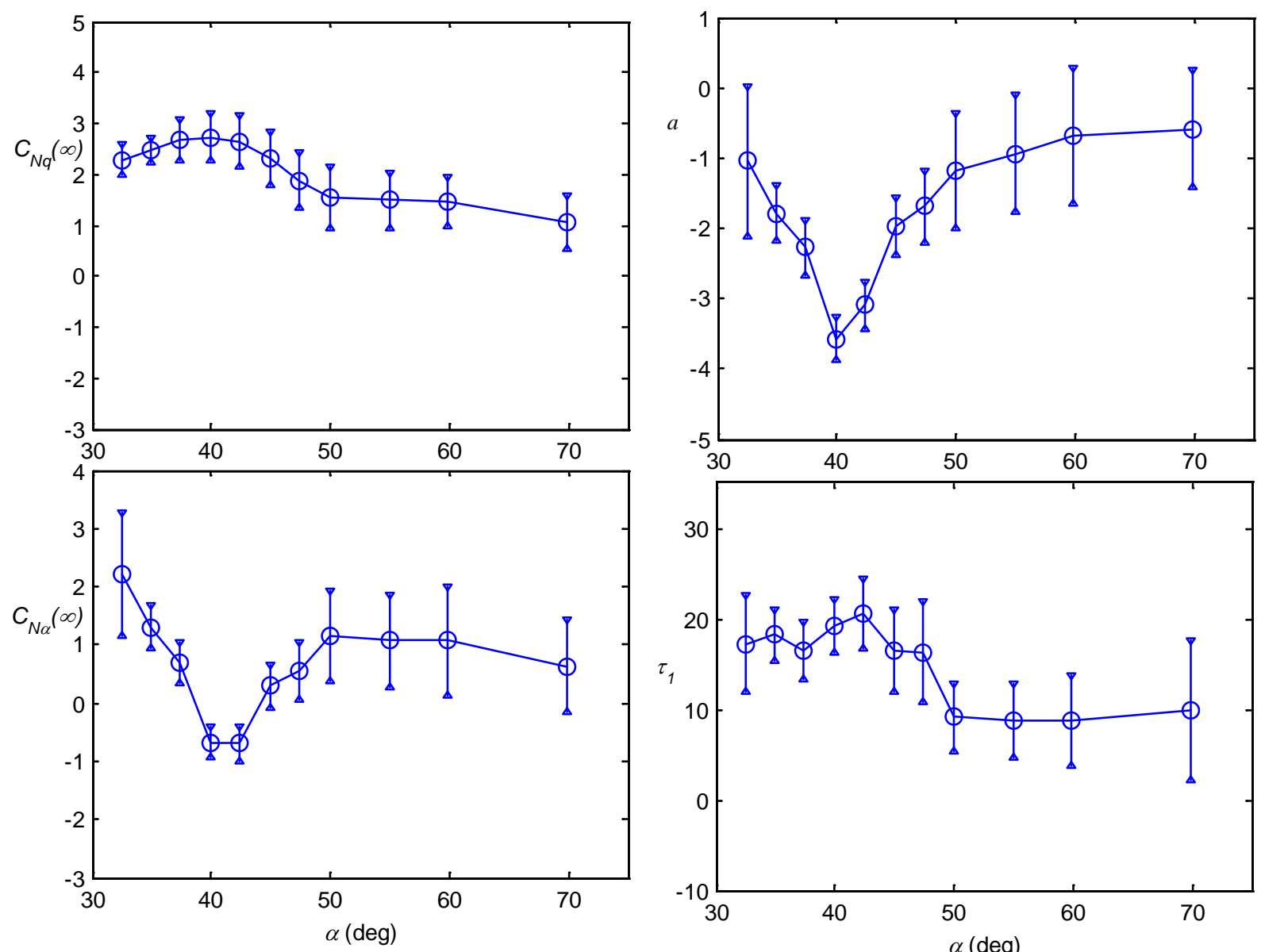

Figure 21a. Normal Force model parameters and $2-\sigma$ bounds, using OE, 2.5\% F-16XL, $\alpha_{A}=5^{\circ}$.

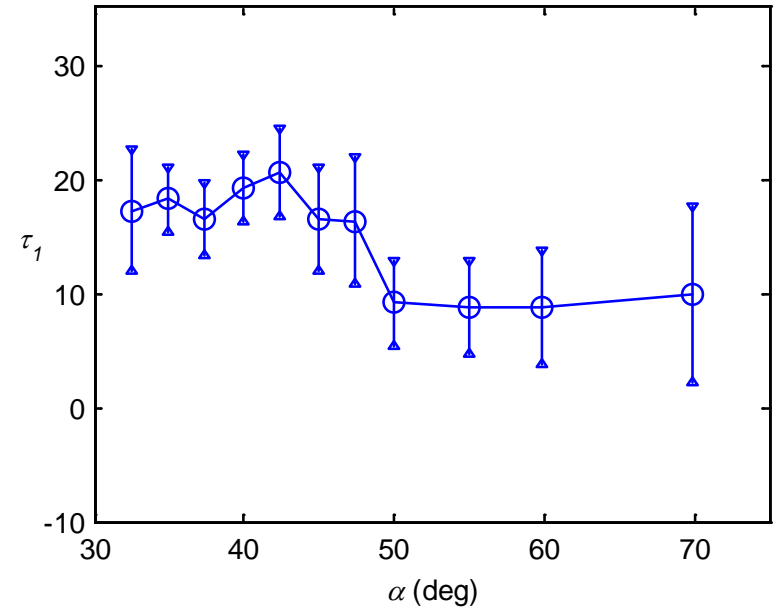

Figure 21b. Normal force model parameters and $2-\sigma$ bounds, using OE, 2.5\% F-16XL, $\alpha_{A}=5^{\circ}$.
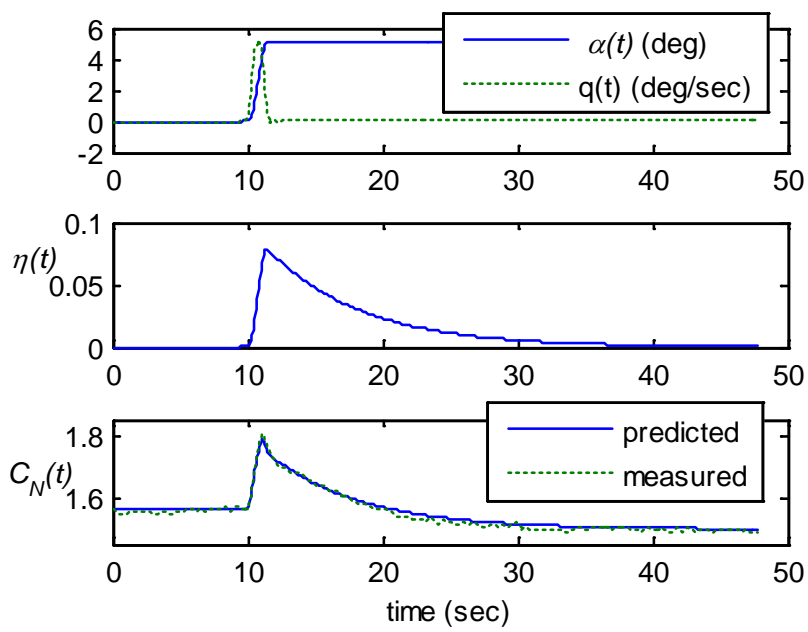

Figure 22. Model validation of $C_{N}$ response to ramp $\left(\alpha_{0}=40^{\circ}-45^{\circ}\right)$, non-dim pitch rate of $0.03,2.5 \% \mathrm{~F}-16 \mathrm{XL}$. 

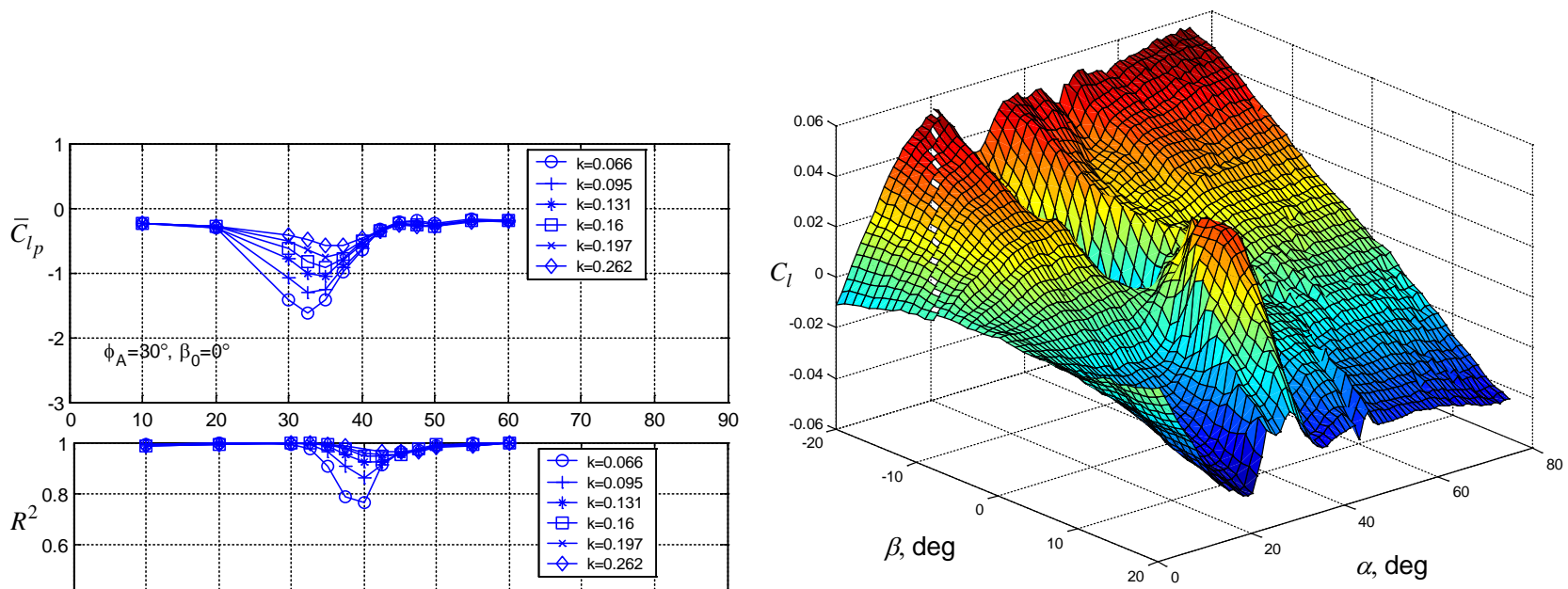

Figure 24. Surface plot of roll moment static measurements, 2.5\% F-16XL.

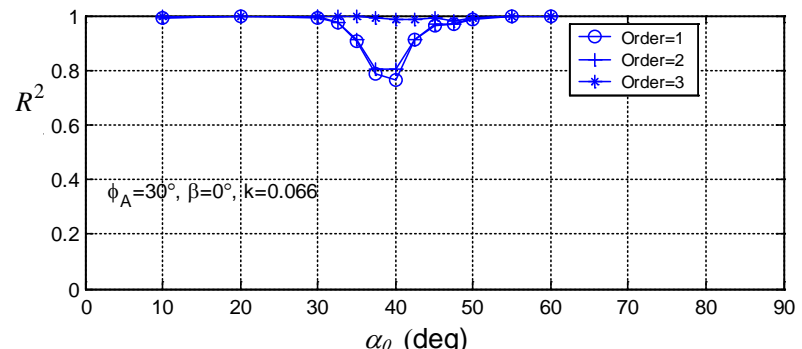

Figure 23. Harmonic Analysis of 2.5\% F-16XL during large amplitude roll oscillations, $\phi_{A}=30^{\circ}$.

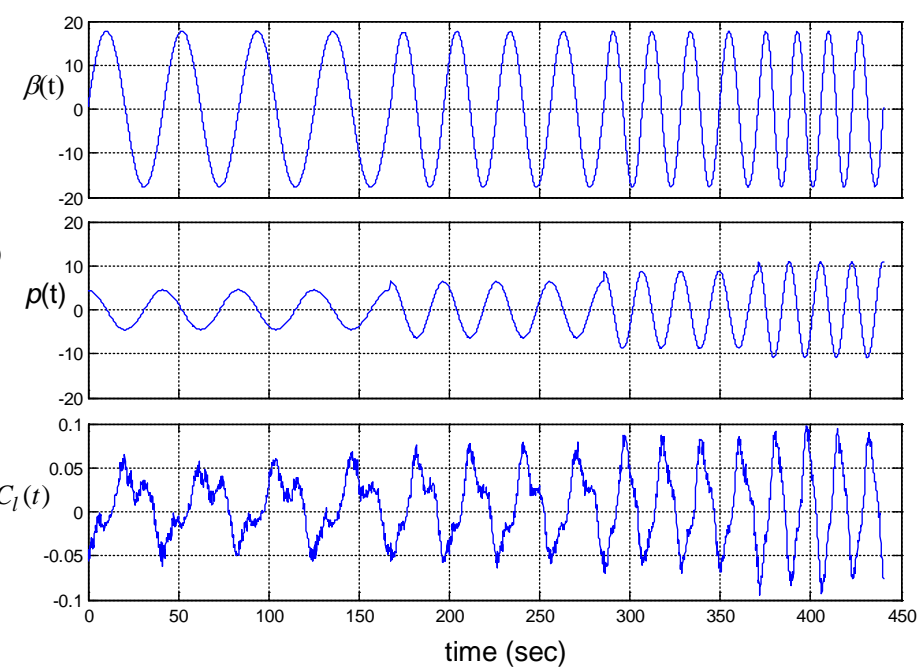

Figure 25. Time histories of the large amplitude oscillatory data at $\alpha_{0}=37.5^{\circ}, \beta_{0}=0^{\circ}$, and four frequencies, $k=[0.066$, 0.095, 0.131, 0.1601, 2.5\% F-16XL. 


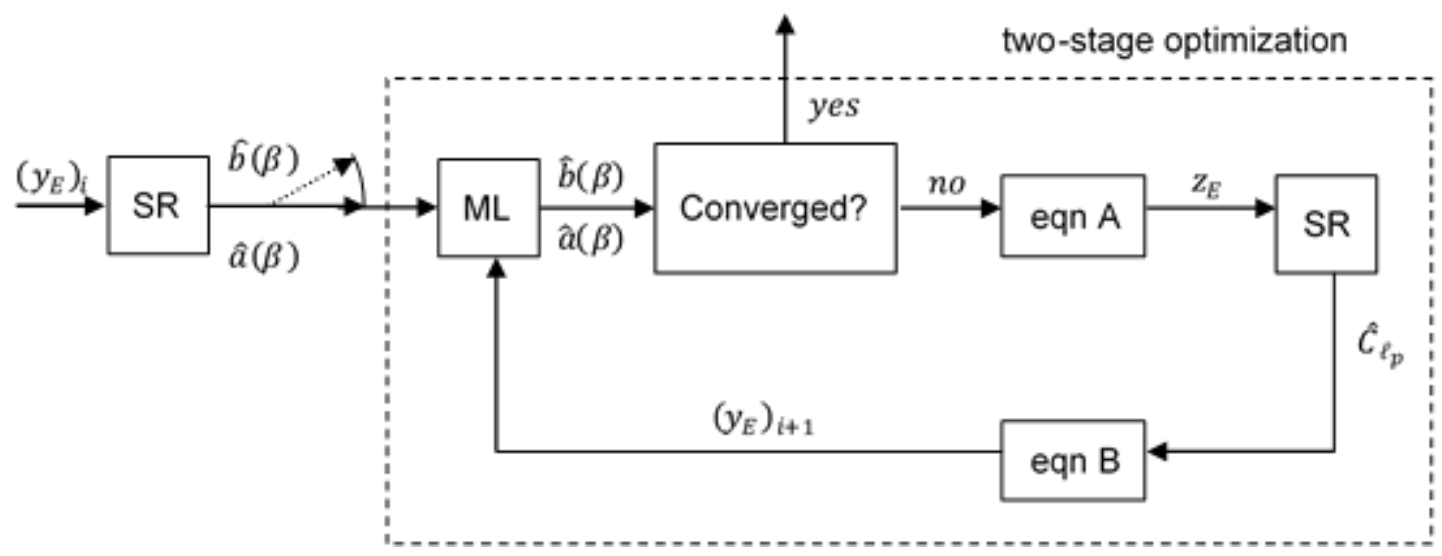

eqn A: $\quad z_{E}(t)=\left(\frac{\ell}{V}\right) C_{a_{p}}\left(\infty ; \alpha_{0}, \beta\right) \mathrm{p}(t)$

eqn B: $\quad y_{E}(t)=C_{a}(t)-C_{a}\left(\infty ; \alpha_{0}, \beta\right)-\left(\frac{\ell}{V}\right) C_{a}\left(\infty ; \alpha_{0}, \beta\right) \mathrm{p}(t)$

Figure 26. Block diagram of model identification procedure using stepwise regression (SR) and an output error (OE) estimation.
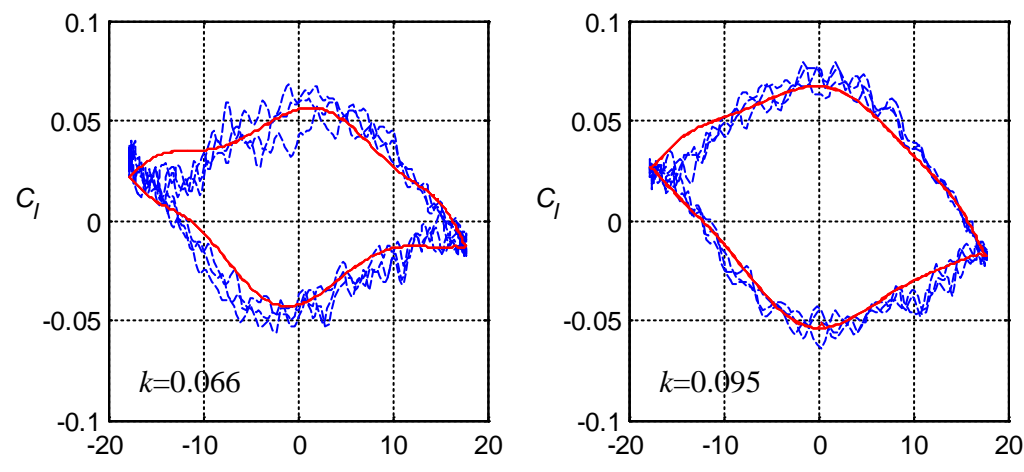

$\beta$, deg

$\beta$, deg
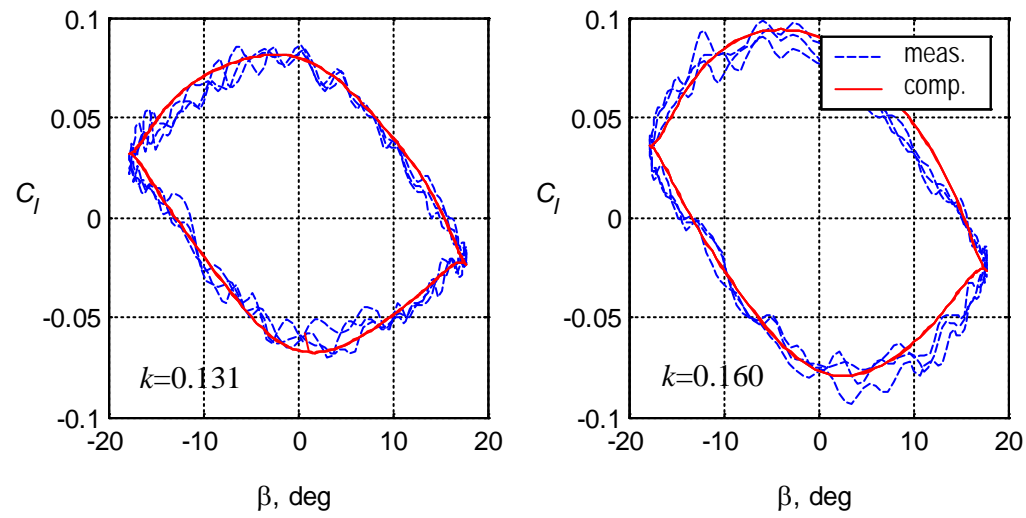

Figure 27. Comparison of measured and computed rolling-moment coefficient from time domain OE method at $\alpha_{0}=37.5^{\circ}, 2.5 \%$ F-16XL. 

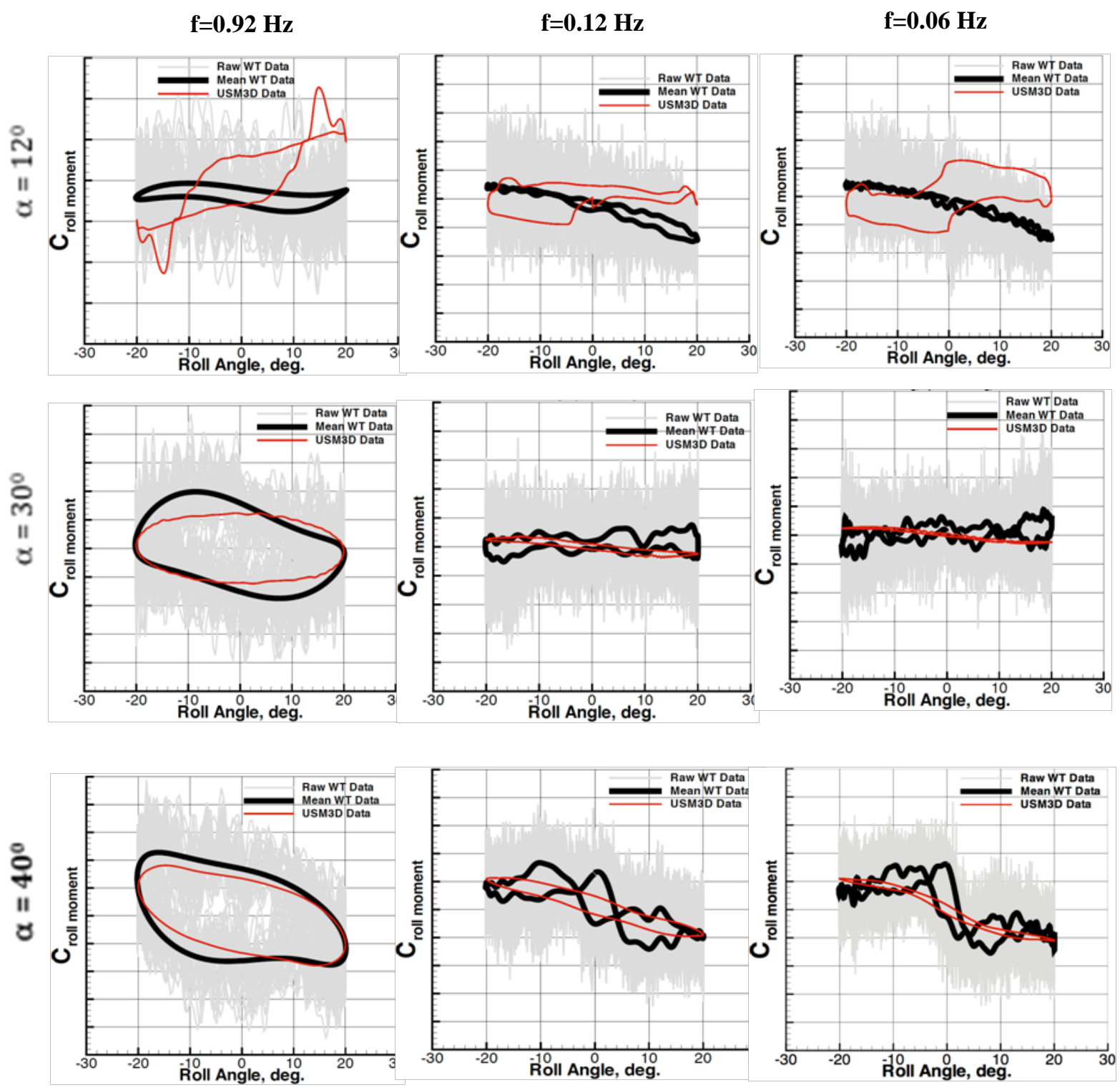

Figure 28. Rolling moment forced oscillations from CFD and wind tunnel (unfiltered and mean-cycle) data, with amplitude $\phi_{A}=20^{\circ}$, for GTM over range of angle of attack and frequency. 


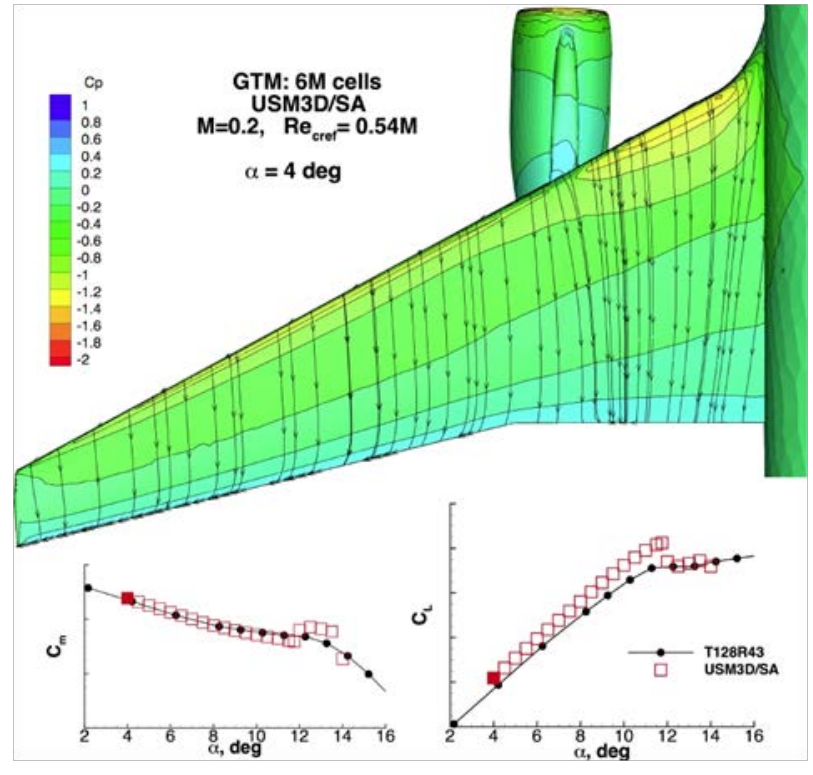

Figure 29a. CFD calculation of streamlines for GTM at $\alpha=4^{\circ}$.

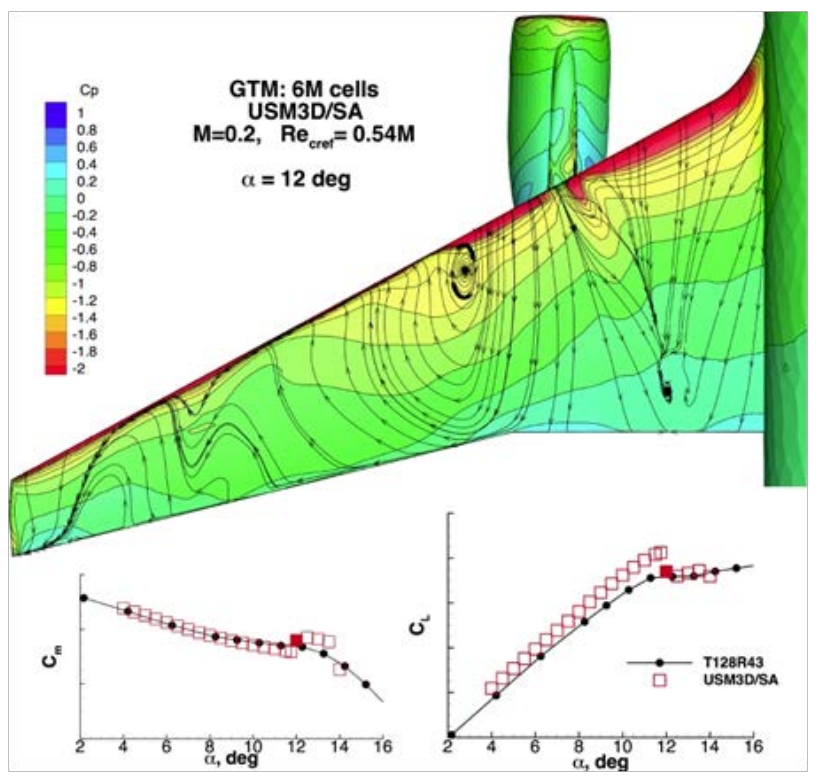

Figure 29c. CFD calculation of streamlines for GTM at $\alpha=12^{\circ}$.

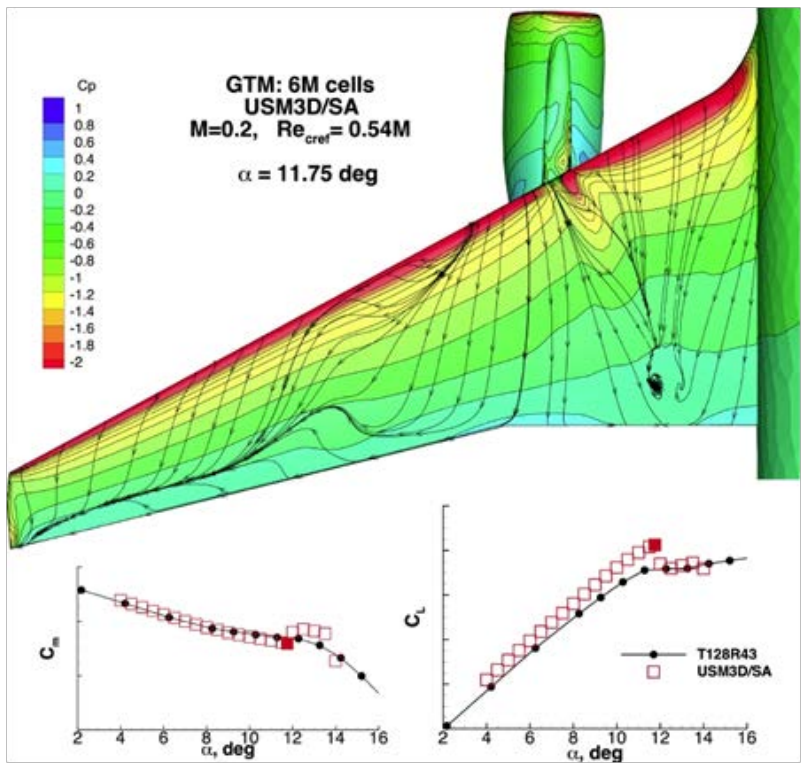

Figure 29b. CFD calculation of streamlines for GTM at $\alpha=11.75^{\circ}$.

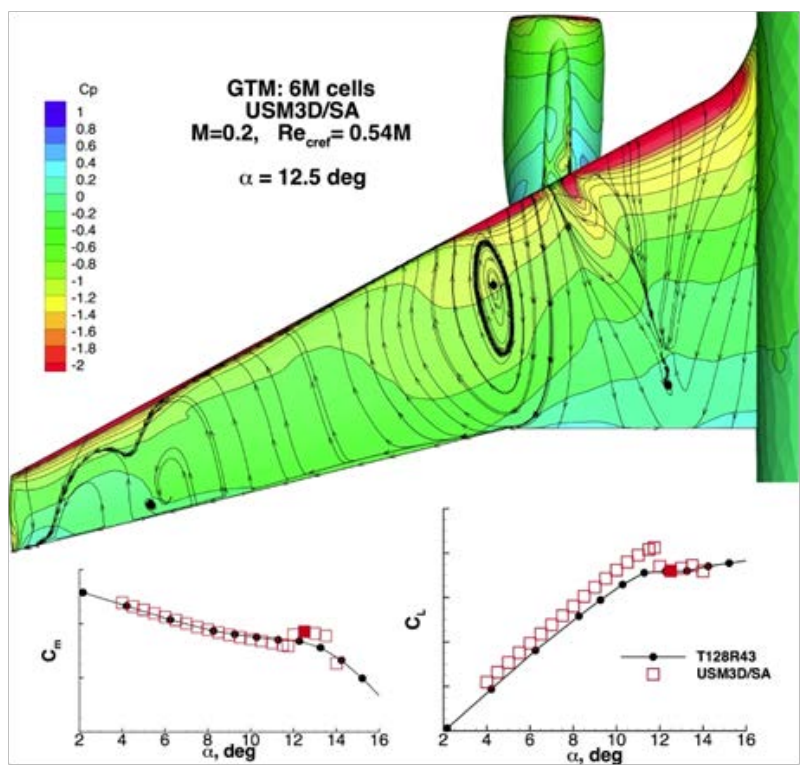

Figure 29d. CFD calculation of streamlines for GTM at $\alpha=12.5^{\circ}$. 

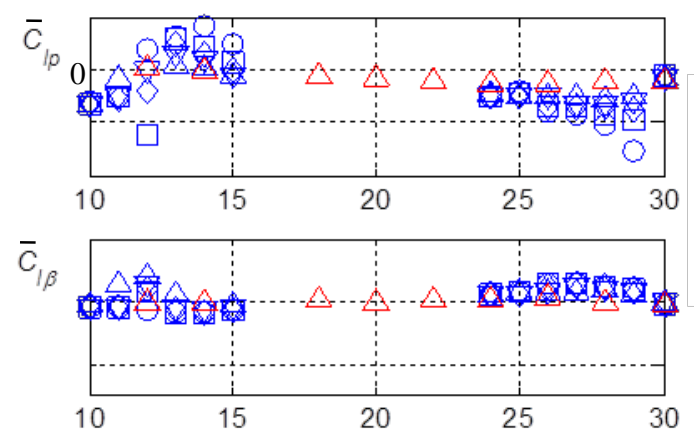

$\mathrm{R}^{2}$

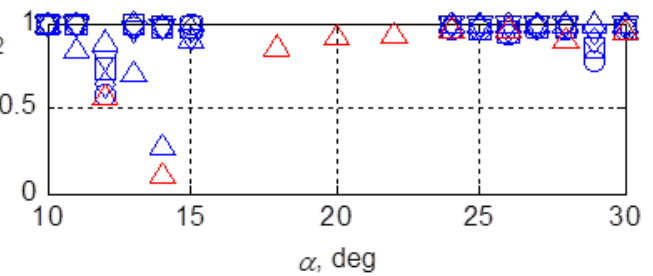

Figure 30. Harmonic analysis for CFD simulated rolling moment coefficient, roll oscillations, $\phi_{A}=20^{\circ}$

(4 $\mathrm{Hz}$ filter applied to wind tunnel data), NASA GTM configuration.

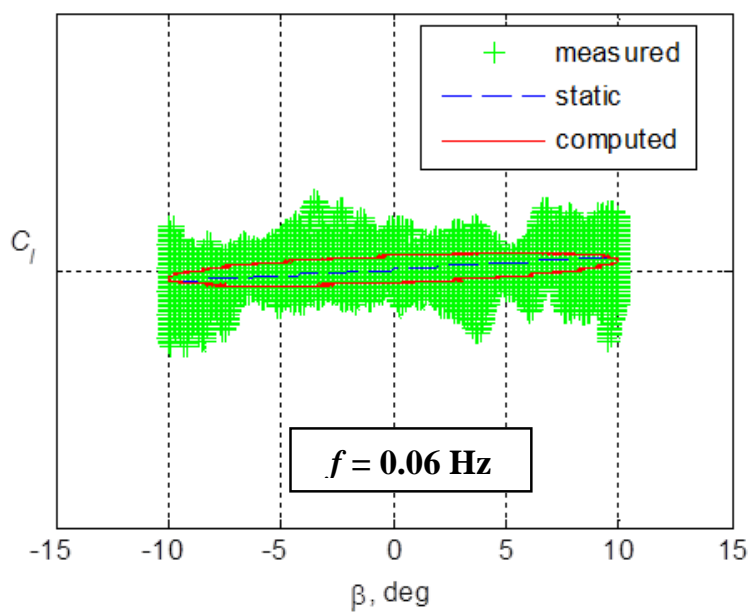

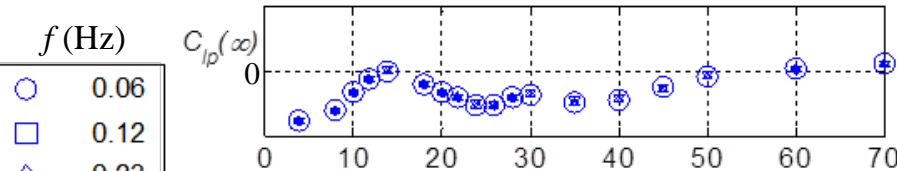

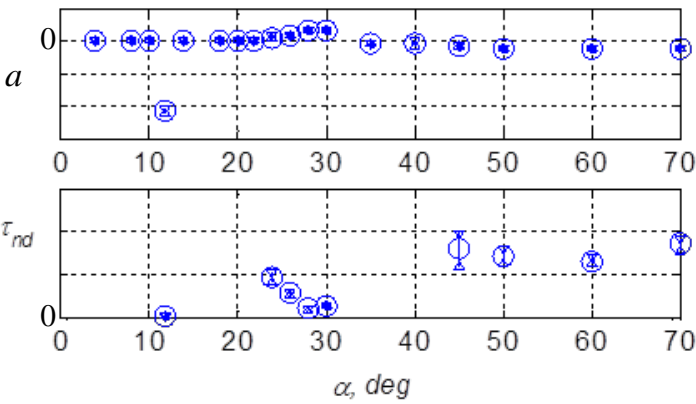

Figure 31. Unsteady model parameters with $2 \sigma$ uncertainty bounds for rolling moment coefficient, $\phi_{A}=20^{\circ}$, NASA GTM configuration.

Figure 32. Unfiltered measurements and unsteady model responses for rolling moment coefficient, at two oscillation frequencies, $\alpha=30^{\circ}, \phi_{A}=20^{\circ}$, NASA GTM configuration. 